\title{
Die Rolle der Luftschadstoffe für die Gesundheit
}

\author{
Eine Replik auf die Expertise der Internationalen Gesellschaft für Umwelt- \\ epidemiologie (ISEE) und der European Respiratory Society (ERS)
}

\section{The Role of Air Pollutants for Health}

\author{
A Reply to the Expert Opinion of the International Society for Environmental \\ Epidemiology (ISEE) and the European Respiratory Society (ERS)
}

Autoren

D. Köhler ${ }^{1}$, M. Hetzel², M. Klingner ${ }^{3}$, T. Koch ${ }^{4}$, S. Ewig ${ }^{5}$, G. Becher ${ }^{6}$, H. Lindemann ${ }^{7}$, T. Voshaar ${ }^{8}$, U. Costabel $^{9}$

Institute

1 Schmallenberg

2 Klinik für Pneumologie, Internistische Intensivmedizin, Beatmungsmedizin und Allgemeine Innere Medizin, Krankenhaus vom Roten Kreuz, Stuttgart

3 Fraunhofer-Institut für Verkehrs- und Infrastruktursysteme IVI, Dresden

4 Leiter des Instituts für Kolbenmaschinen, Karlsruher Institut für Technologie (KIT), Karlsruhe

5 Thoraxzentrum Ruhrgebiet, Kliniken für Pneumologie und Infektiologie, Evangelisches Krankenhaus Herne und Augusta-Kranken-Anstalt Bochum, Herne und Bochum

6 BecherConsult GmbH Bernau

7 Ehemaliger Leiter des Selbständigen Funktionsbereichs für Pneumologie und Allergologie am Zentrum für Kinderheilkunde und Jugendmedizin der Justus-LiebigUniversität Gießen

8 Lungenzentrum Pneumologie, Allergologie, Immunologie, Schlaf-und Beatmungsmedizin. Krankenhaus Bethanien, Moers

9 Zentrum für interstitielle und seltene Lungenerkrankungen, Ruhrlandklinik, Universitätsmedizin Essen

\section{HINWEIS FÜR DIE LESER}

In den letzten Monaten haben Experten und Öffentlichkeit intensiv über Luftschadstoffe diskutiert. Thieme möchte aus diesem Grund maximale Transparenz und beste $\mathrm{Zu}$ gänglichkeit zu Informationen rund um dieses Thema schaffen. Deshalb stellen wir diesen Artikel für die nächste Zeit freizugänglich (free access) zur Verfügung. Um vor dem Hintergrund dieser öffentlichen Diskussion den wissenschaftlich fundierten Diskurs zu fördern, wurde dieser Standpunkt-Artikel - abweichend zu unseren sonstigen Publikationsrichtlinien - nach rein formaler Begutachtung durch die Schriftleitung publiziert (siehe dazu das Editorial von Herrn Professor Schaberg in diesem Heft).
Bibliografie

DOI https://doi.org/10.1055/a-0873-3574 |

Online-Publikation: 16.4.2019 |

Pneumologie 2019; 73: 274-288

(c) Georg Thieme Verlag KG Stuttgart · New York ISSN 0934-8387

Korrespondenzadresse

Prof. Dr. Dieter Köhler, Auf dem Kamp 11,

57392 Schmallenberg

hdieter.koehler@t-online.de

\section{ZUSAMMENFASSUNG}

In Deutschland gibt es regionale Fahrverbote für ältere Dieselfahrzeuge ohne SCR-Katalysator bei Überschreitung der Grenzwerte für $\mathrm{NO}_{2}$. Dies hat zu einer intensiven Diskussion über die Rolle der Luftschadstoffe für die Gesundheit geführt. In der Replik wird dargestellt, dass die Daten zur Wirkung von Stickstoffdioxid $\left(\mathrm{NO}_{2}\right)$ und Feinstaub (PM10 und $\mathrm{PM} 2,5$ ) nicht ausreichen, um die Fahrverbote zu begründen. Für $\mathrm{NO}_{2}$ gibt es passagere Reaktionen bei unbehandelten Asthmatikern ab $500 \mu \mathrm{g} / \mathrm{m}^{3}$. Die deutschen Grenzwerte (Jahresmittelwert $40 \mu \mathrm{g} / \mathrm{m}^{3}$ ) fußen im Wesentlichen auf einer Metaanalyse von 9 Studien aus Innenraumbelastungen wobei nur in 4 Studien $\mathrm{NO}_{2}$ gemessen wurde. In der großen europäischen Escape-Studie von 2014 wurde kein Einfluss von $\mathrm{NO}_{2}$ auf die Mortalität gefunden.

Als Surrogatparameter für andere Schadstoffe ist $\mathrm{NO}_{2}$ ebenfalls nicht mehr geeignet, da seit Einführung der Partikelfilter bei Dieselautos (etwa ab 2000) der KFZ-Anteil am Feinstaub an der Straße unter $10 \%$ liegt. Der Feinstaub besteht im Wesentlichen aus Aufwirbelung von mineralischen, organischen Bodensubstanzen sowie Reifenabrieb und wird am stärksten durch Wetterphänomene, vor allen Dingen durch Sonneneinstrahlung beeinflusst.

Die Grenzwerte für $\mathrm{NO}_{2}$ und Feinstaub werden errechnet aus epidemiologischen Beobachtungsstudien. Es findet sich zumeist eine schwache Assoziation zwischen der Konzentration und zahlreichen Erkrankung sowie der Mortalität. Epidemiologische Beobachtungsstudien erlauben nur die 
Bildung einer Hypothese. Permanente Wiederholungen der Beobachtungsstudien betätigen nur, dass manche gefundenen Phänomene nicht zufällig sind. Eine Kausalität kann daraus nicht abgeleitet werden, da es zahlreiche Erklärungsmodelle neben dem $\mathrm{NO}_{2}$ und Feinstaub gibt. Dazu wären Interventionsstudien im Niedrigdosisbereich sowie Tierexperimente erforderlich. Diese Daten fehlen nahezu komplett bzw. sind, soweit vorhanden, allesamt negativ.

Nie diskutiert wird eine starke Widerlegung der Hypothese der Gefährdung von $\mathrm{NO}_{2}$ und Feinstaub im Grenzwertbereich durch das Inhalationsrauchen. Die Raucher stellen quasi einen inhalationstoxikologischen Großversuch dar. Der Zigarettenrauch enthält sehr hohe Feinstaub-, Stickstoffmonoxid- (NO) und $\mathrm{NO}_{2}$-Konzentrationen, die vom Organismus erstaunlich gut toleriert werden. Das hängt damit zusammen, dass NO ein Naturstoff ist, der in den Zellen oder auch in den Nasennebenhöhlen in z. T. sehr hohen Konzentrationen (über $30000 \mu \mathrm{g} / \mathrm{m}^{3}$ ) vorkommt. Eines der Abbauprodukte von $\mathrm{NO}$ ist $\mathrm{NO}_{2}$, was im Wasser zu Nitrat und Nitrit disproportioniert wird. Ein Teil von $\mathrm{NO}_{2}$ wird zur Synthese von Fettsäuren verwendet.

Zigaretten haben ein Kondensat von ca. 7-10mg. Nimmt man als Vergleich eine lebenslange Dauerbelastung durch Feinstaub und $\mathrm{NO}_{2}$ in den Grenzwertkonzentrationen an, müssten alle Raucher nach wenigen Tagen bis Monaten zahlreiche Erkrankungen entwickeln, die dem Feinstaub und $\mathrm{NO}_{\mathrm{x}}$ angelastet werden. Auch die Mortalität müsste drastisch erhöht sein; nahezu alle Raucher müssten bereits nach 1 packyear verstorben sein. Der Unterschied wird noch größer, wenn man die nachgewiesene Toxizität und Kanzerogenität des Zigarettenrauchs im Vergleich zu dem i.d.R. deutlich weniger gefährlichen Feinstaub an der Straße ins Verhältnis setzt.

\section{ABSTRACT}

In Germany there are regional driving bans on older diesel vehicles without SCR catalytic converters if the limit values for nitrogen dioxide $\left(\mathrm{NO}_{2}\right)$ are exceeded. This has led to an intensive discussion about the effects of air pollutants on human health. The study shows that the data on the effects of $\mathrm{NO}_{2}$ and particulate matter (PM10 and PM2.5) are not sufficient to justify the driving bans.

$\mathrm{NO}_{2}$ above $500 \mu \mathrm{g} / \mathrm{m}^{3}$ trigger temporary reactions in untreated asthmatics The German limit values (annual mean value $40 \mu \mathrm{g} / \mathrm{m}^{3}$ ) are mainly based on a meta-analysis of nine studies on indoor pollution, whereas only four studies measured $\mathrm{NO}_{2}$. In the large European Escape study of 2014, no influence of $\mathrm{NO}_{2}$ on mortality was found.

$\mathrm{NO}_{2}$ is also no longer suitable as a surrogate parameter for other pollutants, as the proportion of particulate matter on the road is below $10 \%$ since the introduction of particulate filters in diesel cars (approximately from 2000). Particulate matter mainly consists of swirling up mineral, organic soil substances and tyre abrasion and is most strongly influenced by weather phenomena, above all by solar radiation.

The limit values for $\mathrm{NO}_{2}$ and particulate matter are calculated from epidemiological observational studies. There is usually a weak association between the concentration of the pollutants and numerous diseases and mortality. On the basis of epidemiological observational studies, hypotheses can be formulated. Repeated observational studies might suggest that some of the observed phenomena are not accidental. A causality cannot be deduced from this, since other factors besides $\mathrm{NO}_{2}$ and particulate matter might also be involved. To exclude these, intervention studies in the low-dose range and animal experiments are needed. Such data are almost completely missing or, as far as available, are all negative.

A strong refutation of the hypothesis of the hazard of $\mathrm{NO}_{2}$ and particulate matter in the limit value range by inhalation smoking is never discussed. The smokers represent quasi an inhalation toxicological large-scale experiment. Cigarette smoke contains very high concentrations of particulate matter, nitrogen monoxide (NO) and $\mathrm{NO}_{2}$, which are surprisingly well tolerated by the organism. This is due to the fact that NO is a natural substance that occurs in the cells or even in the paranasal sinuses in sometimes very high concentrations (over $30,000 \mathrm{\mu g} / \mathrm{m}^{3}$ ). One of the degradation products of $\mathrm{NO}$ is $\mathrm{NO}_{2}$, which, by hydrolytic disproportionation, is converted into nitrate and nitrite. A part of $\mathrm{NO}_{2}$ is used for the synthesis of fatty acids.

Cigarettes have a condensate of about 7-10 mg. Assuming a lifelong continuous exposure to particulate matter and $\mathrm{NO}_{2}$ in the limit concentrations, all smokers should have developed numerous diseases after a few days to months, which are attributed to particulate matter and $\mathrm{NO}_{2}$. The mortality rate should also be drastically higher; almost all smokers should have died after 1 pack year. The difference becomes even greater if one compares the proven toxicity and carcinogenicity of cigarette smoke with the fine dust on the road, which is usually much less dangerous.

\section{Einleitung}

Eine Gruppe deutscher Lungenärzte und Ingenieure unter Federführung von Prof. Dieter Köhler hat über eine Presseerklärung am 23.01.2019 die wissenschaftliche Begründung der EU-weit geltenden Grenzwerte für Stickoxide und Feinstäube sowie Wirksamkeit und Verhältnismäßigkeit von Luftreinhaltemaßnahmen wie Diesel-Fahrverboten in Innenstädten grundsätzlich infrage gestellt. Diese Initiative hat in der Folge ein enormes Echo in öffentlichen Kommunikationsmedien, Politik und wissenschaftlichen Fachkreisen gefunden.

Die ERS hat zusammen mit der ISEE unter dem Titel „Die Rolle der Luftschadstoffe für die Gesundheit“ eine Erwiderung in deutscher Sprache publiziert, in der die Argumente der Initiative als unbegründet zurückgewiesen werden und auf einer gesicherten Evidenz für eine erhöhte Morbidität und Mortalität durch Luftschadstoffe insistiert wird [1]. 
Die vorliegende Publikation stellt dieser Argumentation die wissenschaftlich begründeten Thesen der Initiative deutscher Lungenärzte und Ingenieure entgegen. Sie beantwortet dabei u. a. drei wesentliche Fragen.

- Werden durch Luftschadstoffe im Konzentrationsbereich der Grenzwerte Krankheiten verursacht?

- Sind die geltenden Grenzwerte aus medizinischer Sicht wissenschaftlich begründet?

- Welchen natürlichen und anthropogen verursachten Einflüssen unterliegen die Feinstaub- und Stickoxidkonzentrationen in der Außenluft?

\section{Klarstellungen}

Die Lungenärzte und Ingenieure, die diese Initiative tragen, unterstützen selbstverständlich alle Maßnahmen, die geeignet sind, der Vermeidung von Gesundheitsschäden durch Luftschadstoffe zu dienen.

Die Motivation dieser Initiative ist getragen von der Wahrnehmung eines Missverhältnisses der wissenschaftlichen Datenlage zum potenziellen Einfluss von Luftschadstoffen im aktuellen Bereich der Grenzwerte auf die Gesundheit und der daraus abgeleiteten politischen Maßgaben und richterlichen Urteile.

Die Autoren sehen in diesem Missverhältnis die Gefahren volkswirtschaftlicher und ökologischer Schäden sowie das Risiko einer Beschädigung des Vertrauens in Wissenschaft und Politik. Die Initiative hat keinerlei Interessenskonflikte hinsichtlich umweltpolitischer Zielbestimmungen. Die Autoren verurteilen ausdrücklich den Betrug in Form illegaler Softwarefunktionen durch Teile der Automobilindustrie. Ziel ist einzig, den politisch notwendigen Güterabwägungen auf dem Gebiet der Verkehrspolitik und möglicher sozialer Folgen eine nüchterne interdisziplinäre wissenschaftliche Grundlage zurückzugeben.

Die Autoren dieser Replik sind als wissenschaftlich ausgebildete und tätige Ärzte, Ingenieure und Sachverständige hinreichend kompetent, ihre Befunde und Thesen zu vertreten. Die Auseinandersetzung mit diesen Thesen soll zu einer Versachlichung der Diskussion beitragen.

Die Initiative ist Ausdruck ihres Engagements als Ärzte, Wissenschaftler und Staatsbürger.

\section{Thesen}

1. Die bisher in epidemiologischen Studien gefundenen Assoziationen von Krankheiten durch Exposition auf Luftschadstoffe sind gering im Umfang, inhomogen und inkonsistent. Insgesamt lassen sie eher auf methodische Probleme - insbesondere der Expositionsabschätzung - in diesen Studien schließen.

2. Die aktuell gültigen Grenzwerte für Feinstaub und $\mathrm{NO}_{2}$ sind wissenschaftlich nicht ausreichend begründet.

3. Das Bestreben nach Verbesserung der Luftreinhaltung sollte angesichts dieser Unsicherheiten geleitet sein von einer nachhaltigen Perspektive unter Wahrung der wissenschaftlichen Prinzipien, der Güterabwägung und der Verhältnismäßigkeit.

\section{Werden durch Luftschadstoffe im Konzentrationsbereich der Grenzwerte Krankheiten verursacht?}

Die wissenschaftliche Begründung für einen Zusammenhang zwischen Luftschadstoffen und Krankheiten ergibt sich aus toxikologischen und epidemiologischen Studien.

Die verfügbaren epidemiologischen Studien stammen überwiegend aus den USA und Europa. Die Datenlage wurde durch die amerikanische U.S.EPA (United States Environmental protection agency) zuletzt für Feinstaub im Jahre 2009 [2] und für Stickoxide 2016 [3] in einem Dokument zusammengefasst und bewertet.

Das größte Forschungsprojekt aus Europa ist die „European Study of Cohorts for Air Pollution Effects (ESCAPE)-Studie“ (2008-2012; [4]). Ziel von ESCAPE ist die weitere Abschätzung der Wirkung von Luftschadstoffen für bereits vorliegende europäische Kohortenstudien. Die publizierten Daten sind demnach alle Metaanalysen von Einzelstudien, da die Daten der Kohorten nicht zusammengelegt („gepoolt“) werden konnten.

Die ISEE- und ERS-Expertise [1] hat sich in ihrer Argumentation im Wesentlichen auf diese Dokumente bezogen.

Dabei werden in der Expertise zahlreiche gesundheitliche Auswirkungen als „kausal etablierte Wirkung“ angesehen.

Eine kritische Darstellung der Limitationen dieser Studien, insbesondere der Unsicherheiten bei der Expositionsabschätzung, fehlt.

\section{Kritik an der Darstellung der Studienlage}

Die in der Expertise dargestellten Ergebnisse stimmen teilweise nicht mit dem aktuellen Forschungsstand überein und sind mitunter selektiv ausgewählt.

Zum Einfluss von Feinstaub auf die kardiovaskuläre Mortalität werden ältere Arbeiten angeführt [2], obwohl die Metaanalyse mit den aktuell größten Probandenzahlen aller europäischen Studien von 2014 keinen Zusammenhang mehr gefunden hat [5]. Diese Arbeit wird in der Expertise nicht zitiert.

Keinen Einfluss auf die kardiovaskuläre Mortalität hatte auch die Metaanalyse von Beelen et al. [6] ergeben, die in der Expertise zwar zitiert wird, jedoch in anderem Zusammenhang. Sie wird als Bestätigung des negativen Einflusses von $\mathrm{NO}_{2}$ auf Herz-Kreislauf-Erkrankungen erwähnt. Für Herz-Kreislauf-Erkrankungen war in dieser Metaanalyse aber für $\mathrm{NO}_{2}$ kein erhöhtes relatives Risiko gefunden worden.

In der Expertise werden vier weitere Arbeiten [7 - 10] zitiert, um auf einen positiven Zusammenhang von $\mathrm{NO}_{2}$ und HerzKreislauf-Erkrankungen hinzuweisen. Die größte und hochrangig publizierte Metaanalyse der europäischen ESCAPE-Studiendaten [11] wird hingegen nicht angeführt, obwohl sie keinen Zusammenhang zwischen $\mathrm{NO}_{2}$ und der Gesamtmortalität gezeigt hat.

Diese Metaanalyse [11] wird in der Expertise nur in der Zusammenfassung zitiert, und zwar als Beweis für Auswirkungen von PM2,5 unterhalb des aktuellen Grenzwertes. Eine weitere Analyse der ESCAPE-Daten bzgl. der Zusammensetzung von PM2,5 zeigte in dieser Metaanalyse nur für PM2,5sulfur eine 
signifikante Assoziation mit der Gesamtmortalität [12]. PM2,5sulfur jedoch entsteht aus $\mathrm{SO}_{2}$, das in den letzten Jahren praktisch keine Rolle mehr spielt, denn es ist in Deutschland seit 1990 um über $90 \%$ gesunken [13].

\section{Methodenkritik epidemiologischer Studien}

Nahezu alle bisherigen epidemiologischen Studien zu Luftschadstoffen leiden unter einer Reihe von methodischen Problemen, Unsicherheiten und fehlenden Daten. Dies erhöht gerade bei vielen möglichen Umweltfaktoren und multiplen Testen das Konfidenzintervall und damit das Risiko von Fehlinterpretationen [14]. Effekte der Gesamtexposition auf Luftschadstoffe wurden sehr häufig als „suggestive evidence“ (z. B. [15]), „strongly supports“ (z. B. [9]) oder „contributed“ (z. B. [16]) auf die Zielgröße bewertet, trotz der immer nur schwachen Assoziationen, die mitunter nicht die Signifikanzschwelle erreichten, sofern diese angegeben wurde (z. B. [15]).

In den epidemiologischen Studien, so auch in den ESCAPEStudien, gibt es keine, wie typisch in der Arbeitsmedizin, personenbezogene Schadstofferfassung. Bei den Luftmessungen wird häufiger die Nachweisgrenze nicht erreicht [11].

Weiterhin waren die Probanden in den ESCAPE-Studien oft noch relativ jung. Ein Aufenthaltsmuster wurde nicht erfasst und ist auch retrospektiv schwer zu erfassen. Berufliche Expositionen wurden oft nicht hinreichend erfasst, denn hier können weitaus höhere Expositionen vorliegen. Es finden sich keine verlässlichen Daten zur Aufenthaltsdauer in Innenräumen. Messungen von Innenraumimmissionen erfolgten nicht.

Folgeuntersuchungen fehlen oft bzw. werden vereinzelt nur in Untergruppen durchgeführt. Damit fehlen wichtige Informationen über Änderungen der starken Risikofaktoren wie Rauchen, Alkohol, Bewegungsmangel, Übergewicht usw. im Zeitverlauf. Bei ESCAPE lagen meist ca. 14 Jahre zwischen Untersuchung und Ereignis (Mortalität, Schlaganfall, Tumor usw.) im Follow-up [11].

Wesentliche Kritiken an der ESCAPE-Studie sind bisher nicht widerlegt worden, so bspw., dass sich keine konsistenten Ergebnisse für bestimmte Schadstoffe oder ihre Kombinationen zwischen diesen Studien ergeben hätten [17]. Ähnliches gilt auch für andere epidemiologische Studien, wo insbesondere bemängelt wird, dass die Innenraumbelastung nicht adäquat berücksichtigt wurde [18].

Ebenso wenig schlüssig ist die fehlende Assoziation mit kardiovaskulärer [5] im Gegensatz zur gefundenen Assoziation mit zerebrovaskulärer Mortalität [19] mit PM2,5, wo doch die Atherosklerose den gemeinsamen Mechanismus darstellen müsste. Die gleiche Inkonsistenz fand sich auch für $\mathrm{NO}_{2}$; hier war $\mathrm{NO}_{2}$ mit einer reduzierten zerebrovaskulären Mortalität im Vergleich zur kardiovaskulären assoziiert [10].

Darüber hinaus werden mögliche Assoziationen fälschlich als kausale Zusammenhänge postuliert. Assoziationen oder Korrelationen in Kohortenstudien dienen nur zur Hypothesenbildung; sie beweisen jedoch nie einen kausalen Zusammenhang [20-22].

Besonders deutlich wird dieses Problem bspw., wenn eine Risikoerhöhung aus einer epidemiologischen Beobachtungsstudie für ein Medikament [23] durch zwei randomisiert kon- trollierte Studien mit bewusst ausgewählten ähnlichen Patientengruppen überprüft wird [24,25]. Die in den epidemiologischen Beobachtungsstudien gefundenen, teilweise hohen Risikoerhöhungen verschwanden in diesen randomisiert kontrollierten Studien ausnahmslos.

Wesentliche sozioökonomische, soziodemografische Faktoren sowie das Gesundheitsbewusstsein bestimmen maßgeblich die Lebenserwartung [26-32]. Viele davon wurden nicht oder können auch gar nicht verlässlich gemessen werden. Allein die fehlende Adhärenz zu einer Therapie (unabhängig von der Wirksamkeit der Therapie) kann die Mortalität bereits mehr als verdoppeln [33]. Eine solche Gesundheitseinstellung ist durch Fragebögen nicht zu erfassen.

Die ISEE- und ERS-Expertise weist darauf hin, dass in allen relevanten Studien eine sorgfältige Adjustierung erfolgt sei. Die Anzahl von Dutzenden relevanter Störfaktoren in Kombination mit mikro- und makrospezifischen Einflussfaktoren lässt mit den erfassten probandenspezifischen Daten eine mathematisch präzise Zuordnung der einzelnen Wirkmechanismen hingegen nicht zu [14]. Die Methoden zur Adjustierung sind zu unterkomplex und könnten wesentliche Effekte nicht berücksichtigen, insbesondere auch, weil viele Confounder 10- bis 100-fach und mehr über der Wirkung der Messgröße liegen [34 -36]. Das gilt insbesondere für Zigarettenrauchen [37], Alkohol, Übergewicht, Bewegungsmangel und Komorbiditäten, die im Verlauf auftreten und dann nicht mehr erfasst werden [38].

Schließlich liegen allen epidemiologischen Studien Modellrechnungen zugrunde, die von Studie zu Studie sehr unterschiedlich gewählt sind. Je nach Modellrechnung kann das Ergebnis erheblich variieren, was insbesondere Metaanalysen erschwert.

\section{Sind die Grenzwerte wissenschaftlich begründet? \\ Welchen natürlichen und anthropogen verursachten Einflüssen unterliegen die Feinstaub- und Stickoxidkonzentrationen in der Außenluft?}

Emission = Stoffaustrag einer Quelle (Gramm/Zeit oder Weg)

Immission $=($ Schad-) Stoffkonzentration in der Luft $\left(\mu \mathrm{g} / \mathrm{m}^{3}\right)$, definitionsgemäß an einer Messstelle*

\section{Feinstaub}

In der EU-Richtlinie 2008/50/EC zur Luftqualität und ihrer Verbesserung in Europa (in deutsches Recht umgesetzt mit der 39. Bundes-Immissionsschutz-Verordnung [39. BImSchV]) wur-

\footnotetext{
* Die Immission von Feinstaub und $\mathrm{NO}_{\mathrm{x}}$ nimmt etwa proportional zur Entfernung von der Quelle ab.
} 
de der Grenzwert für Feinstaub wie folgt festgesetzt: Der Tagesgrenzwert für PM10 beträgt $50 \mu \mathrm{g} / \mathrm{m}^{3}$ und darf nicht öfter als 35-mal im Jahr überschritten werden. Der zulässige Jahresmittelwert beträgt $40 \mu \mathrm{g} / \mathrm{m}^{3}$. Seit 2015 gilt für PM2,5 $25 \mu \mathrm{g} /$ $\mathrm{m}^{3}$ im Jahres-Mittelwert; dieser Wert ist deutlich höher als der von der WHO vorgeschlagene Wert von $10 \mu \mathrm{g} / \mathrm{m}^{3}$. Für die Zukunft sind auch niedrigere Werte in Deutschland ins Auge gefasst worden.

In den USA wurde der PM10-Grenzwert von $50 \mu \mathrm{g} / \mathrm{m}^{3}$ für den Jahresmittelwert bereits 2006 aufgrund fehlender Nachweise für gesundheitliche Risiken bei Langzeitexposition aufgehoben [39].

Auch der in den USA geltende Grenzwert für den 24-StundenMittelwert für PM10 ist mit $150 \mu \mathrm{g} / \mathrm{m}^{3}$, der höchstens einmal pro Jahr im 3-jahres-Durchschnitt überschritten werden darf, deutlich moderater gefasst als in den EU-Richtlinien. Niedrigere Grenzwertregelungen gelten hingegen für den PM2,5-3 Jahres-Mittelwert mit $15 \mu \mathrm{g} / \mathrm{m}^{3}$. Zusätzlich ist der Mittelwert in der 98. Perzentile der 24-Stunden-Werte dreier Jahre von $35 \mu \mathrm{g} / \mathrm{m}^{3}$ einzuhalten [40].

Detaillierte Analysen der umfangreichen Messdaten aus den Luftmessnetzen verschiedener Bundesländer haben bekannte Zusammenhänge zur Wechselwirkung natürlicher und menschlich, d.h. anthropogen bedingter Feinstaubquellen bestätigt und Grundlagen für neue Erkenntnisse gelegt, die die Grenzwertregelungen zu Feinstaub infrage stellen.

Feinstaub entstammt heute zu einem weit geringeren Anteil aus anthropogenen Quellen als bisher angenommen. Die satellitengestützten Messungen der globalen PM2,5-Belastung dokumentieren, dass die großen natürlichen Feinstaubquellen in den Wüstengebieten zu finden sind und dass die Intensität der Sonneneinstrahlung bspw. in der Äquatorregion einen wesentlichen Einfluss auf die globale Verteilung der PM2,5-Konzentrationen hat $[41,42]$. So treten auch in Süd- und Mitteleuropa deutlich höhere Feinstaubbelastungen auf als in Nordeuropa. Unsere Einflussmöglichkeiten auf diese Feinstaubbelastung, insbesondere auf die Überschreitung der Tagesgrenzwerte, sind äußerst begrenzt [43].

Denn es sind in erster Linie Wetterphänomene, die in der unteren Atmosphärenschicht extreme Schwankungen und v.a. Spitzenbelastungen verursachen [44-46].

Episoden hoher Feinstaubkonzentrationen mit ganz unterschiedlicher Zusammensetzung treten bspw. nicht nur in urbanen Zentren, sondern auch in ländlichen Gebieten und abgelegenen Alpenregionen fernab von Verkehrsströmen und menschlichen Siedlungen auf.

V.a. austauscharme Inversionswetterlagen verursachen diese hohen Konzentrationen. Industrieabgase und der Hausbrand tragen dann in erheblich höherem Maße zu den Feinstaubkonzentrationen in Bodennähe bei als der Verkehr. Niedrige Mischungsschichthöhen und fehlende Vertikalströmung führen darüber hinaus zu einem konzentrierten Aufspeichern des Feinstaubs in der Luft und damit zu besonders hohen Feinstaubkonzentrationen $[44,46]$.

Feinstaub wird durch viele meteorologische Faktoren beeinflusst. Regenereignisse senken die Feinstaubkonzentration und binden Feinstaub am Boden. In diesen Phasen lässt sich der natürliche vom verkehrsbedingten Feinstaub gut differenzieren. In niederschlagslosen Zeiten hingegen steigt die Feinstaubkonzentration unabhängig von der Intensität anthropogener Feinstaubquellen kontinuierlich zwischen 3 und $5 \mu \mathrm{g} / \mathrm{m}^{3}$ pro Tag [44-46].

Wind vermindert einerseits die Feinstaubkonzentration, wirbelt bei höherer Windgeschwindigkeit jedoch auch Feinstaub in Konzentrationsanteilen von bis zu $5 \mu \mathrm{g} / \mathrm{m}^{3}$ vom Boden auf. Bisher weitgehend unberücksichtigt blieb die Wirkung der Sonneneinstrahlung. Allein durch intensive Sonneneinwirkung kann sich die Feinstaubkonzentration um bis zu $40 \mu \mathrm{g} / \mathrm{m}^{3}$ und mehr erhöhen [44-46]. Mit jeder Bewegung wird Feinstaub aufgewirbelt. Hochgeschwindigkeitszüge, U-Bahnen, Busse, Radfahrer, Fußgänger: Alle wirbeln Feinstaub auf. Diese bewirken im eigentlichen Sinn keine Emissionen, aber letztendlich beeinflusst dies die lokale Feinstaubkonzentration nicht unerheblich.

Meteorologische Faktoren wie Niederschlag, Sonneneinstrahlung und Luftschichtung beeinflussen die Feinstaubkonzentrationen großräumig und mit teilweise über Tage anhaltender Nachwirkung. Diese liegen im Vergleich zu dem Eintrag durch den Verkehr i.d. R. deutlich über $80 \%$ [44-47].

Verkehrsintensität und Feinstaubkonzentrationen weisen im Mittel einen sehr ähnlichen Tagesgang auf. Dies führt zu dem weit verbreiteten Missverständnis, dass v.a. der Verkehr den Tagesgang des Feinstaubs verursacht.

Bisher unbeachtet blieb, dass der Tagesgang des Feinstaubs der Intensität der Sonneneinstrahlung folgt und nicht dem Tagesgang des Verkehrsaufkommens [47].

Noch vor rund 30 Jahren war die Immissionssituation eine andere. Die Abgase waren deutlich toxischer $[48,49]$. $\mathrm{NO}_{\mathrm{x}}$ und Rußemissionen standen in unmittelbarer Wechselwirkung, da dieselmotorische Antriebe beide Emissionskomponenten nicht abgasnachbehandlungsseitig reduzierten. Erst mit der flächendeckenden Einführung des Partikelfilters in den 2000er-Jahren und der Einführung der Stickoxidabgasnachbehandlung änderte sich das Bild. Als Konsequenz dieser Entwicklung ist heute keine relevante Korrelation mehr zwischen Rußpartikeln an der Straße und $\mathrm{NO}_{\mathrm{x}}$ gegeben $[50,51]$.

Es wird nicht bestritten, dass eine höhere Konzentration an Feinstaub vor allen Dingen in Verbindung mit Schwefeldioxid zu einer Exazerbation von Atemwegserkrankungen führen kann. Dauerhaft höhere Konzentrationen können auch eine chronische Bronchitis verursachen. Ein deutlich erhöhtes Risiko (RR $2-4$ ) ist insbesondere in älteren Untersuchungen bei offenen Feuerstellen festgestellt worden [52-54]. Auch fand sich früher in Osteuropa eine höhere Inzidenz an chronischer Bronchitis, was u.a. auf die Feinstaub- und $\mathrm{SO}_{2}$-Belastung durch Braunkohle zurückgeführt wurde [55,56]. Dies gilt auch heute in Regionen mit hoher Belastung [57]. Die Asthmainzidenz war hingegen niedriger als im Westen [58].

Zudem liegen überzeugende tierexperimentelle Daten zur Entwicklung einer chronischen Bronchitis durch höhere Feinstaubkonzentrationen vor [59]. Dabei wurden meist Dosen von ca. $500-1000 \mu \mathrm{g} / \mathrm{m}^{3}$ Feinstaub, oft zusammen mit $\mathrm{SO}_{2}$, aus unterschiedlichen Quellen verwendet [60]. Entsprechende tierexperimentelle Daten mit einer Exposition auf die heute viel niedrigeren Feinstaubkonzentrationen liegen nicht vor. 
Die Verursachung der chronischen Bronchitis ist auch von beruflicher Aerosolbelastung bekannt, wenn diese auch ungleich höher liegt als der aktuelle Grenzwert. Deswegen wurden dazu auch die Berufserkrankung BK 4302 (durch chemisch-irritativ oder toxisch wirkende Stoffe verursachte obstruktive Atemwegserkrankungen) und die BK 4111 (Chronische obstruktive Bronchitis oder Emphysem von Bergleuten unter Tage im Steinkohlenbergbau bei Nachweis der Einwirkung einer kumulativen Dosis von i.d.R. 100 Feinstaubjahren $\left[\left(\mathrm{mg}\left(\mathrm{m}^{3}\right) \times\right.\right.$ Jahre $\left.]\right)$ eingeführt [61]. Die 100 Feinstaubjahre haben Bergleute damals schnell erreicht, denn die Feinstaubkonzentrationen unter Tage waren vor 1990 noch sehr hoch, im Mittel bei $5-7 \mathrm{mg} / \mathrm{m}^{3}[62$, 63].

Wenn man die Deposition von $100 \mathrm{~g}$ in der Lunge bei Erreichung der BK 4111 mit der gesamten Feinstaubbelastung $(6,7 \mathrm{~g}$ in $70 \mathrm{~J})$ an der Neckartormessstelle (s. A Abb. 1) vergleicht, dann müsste ein Mensch dort 1045 Jahre stehen, bis er die gleiche Menge inhaliert hat. Vergleicht man gar nur mit dem verkehrsbedingten Anteil (1,4 g), wären das dann 5000 Jahre.

Durch die Clearancemechanismen ist die Gesamtdeposition natürlich geringer; das gilt aber für alle Schadstoffbelastungen [64-66].

Zudem wird häufig vergessen, dass die Innenraumbelastung für Feinstaub sich gravierend von der im Freien unterscheidet. [67 - 70]. In öffentlichen Einrichtungen wie Schulen wurden in Deutschland maximal bis zu $1000 \mu \mathrm{g} / \mathrm{m}^{3}$ PM10 gemessen [71].

\section{Stickoxide}

In derselben EU-Richtlinie 2008/50/EC zur Luftqualität und ihrer Verbesserung in Europa wurde der Grenzwert für $\mathrm{NO}_{2}$ auf $40 \mu \mathrm{g} / \mathrm{m}^{3}$ festgesetzt [72]. In der Begründung wird auf eine WHO-Leitlinie von 2000 und 2005 verwiesen [73, 74]. In dieser wird jedoch ausdrücklich festgehalten, dass ein gut begründeter Grenzwert aus den verfügbaren Studien nicht abgeleitet werden konnte, vielmehr wird auf die Environmental Health Criteria 188 von 1997 verwiesen [75]. Dieses Dokument legt auf der Basis einer Belastung von $15 \mu \mathrm{g} / \mathrm{m}^{3}$ und der Tatsache, dass gesundheitliche Schädigungen ab $28,2 \mu \mathrm{g} / \mathrm{m}^{3}$ additiv auftreten können, einen Grenzwert von $40 \mu \mathrm{g} / \mathrm{m}^{3}$ fest.

Dieser Wert basiert auf einer Metaanalyse von neun Studien aus unterschiedlichen Ländern mit dem Endpunkt „Symptome der unteren Atemwege“ und „Erkrankungen bei Kindern unter 12 Jahren“ [76], wobei nur Innenraumbelastungen, meist durch Gasöfen, berücksichtigt wurden. Nur in vier dieser Studien wurde die tatsächliche Exposition gegenüber Stickoxiden gemessen.

In der großen Metaanalyse des ESCAPE-Projektes von 2014 [11] wurde kein Effekt von $\mathrm{NO}_{2}$ auf die Mortalität gefunden.

Zudem reflektieren Außenluftmessungen mit stationären Geräten nicht die persönliche Belastung [77]. Nicht berücksichtigt wird in epidemiologischen Studien, dass sich in Innenräumen die $\mathrm{NO}_{2}$-Konzentration im Vergleich zur Straße nochmals um ca. die Hälfte halbiert, wenn keine $\mathrm{NO}_{2}$-Quellen aus dem Innenraum hinzukommen (Kerzen, Kamin, Gasherd, Rauchen) $[67,78,79]$.

Untersuchungen am Menschen haben gezeigt, dass gesunde Probanden auf eine Exposition von $\mathrm{NO}_{2}$ bis zu einer Konzentra- tion von $4000 \mu \mathrm{g} / \mathrm{m}^{3}$ keine akuten Symptome entwickeln [80]. Asthmatiker zeigen ab einer Konzentration von ca. $500 \mu \mathrm{g} / \mathrm{m}^{3}$ mitunter eine Veränderung bzw. Triggerung der Hyperreagibilität [81-83]. Für die Entstehung eines Asthmas fanden sich keine Hinweise $[81,84]$. Naturgemäß kann eine Reaktion auch auf geringe Konzentrationen nicht ausgeschlossen werden; Asthmatiker können zudem auf jedwede Exposition von Reizstoffen, einschließlich physiologischer Kochsalzlösung oder Parfüm, in niedriger Konzentration reagieren. Es erscheint jedoch sehr unwahrscheinlich, dass zehnfach niedrigere Konzentrationen chronische Lungenerkrankungen bewirken können. Immerhin rauchen mind. 15-30\% der Asthmatiker [85, 86].

Im Hauptstrom des Zigarettenrauchs entstehen beim Rauchen Konzentrationen von bis zu $1000000 \mu \mathrm{g} / \mathrm{m}^{3}$ NO und sicher auch über $50000 \mu \mathrm{g} / \mathrm{m}^{3} \mathrm{NO}_{2}$ [87-89]. Die Asthmatiker vertragen diese hohen $\mathrm{NO}_{\mathrm{x}}$-Konzentrationen wahrscheinlich nur, weil sie den Hauptstrom aus der Zigarette (Ansaugvolumen) durch Nebenluft verdünnen. Damit reduzieren sich die Konzentrationen ca. um den Faktor 20 - 50.

Stickoxide sind wichtige Biomoleküle, die auch im Körper gebildet werden können. Im Jahr 1988 erhielten Robert Furchgott, Louis Ignarro und Ferrid Murad den Nobelpreis für Medizin für die Rolle der Stickoxide als „signalling molecules“. NO wird im Körper für unterschiedliche Zwecke, darunter Signaltransduktion und Vasodilatation, synthetisiert, da es als Gas sofort durch die Zellwand penetrieren kann [90-92]. NO ist heute ein zugelassenes Medikament zu Vasodilatation der Lungenkapillaren bei beatmeten Patienten.

NO findet sich auch im Exhalat, insbesondere aus der Nase (dabei werden bei Gesunden Konzentrationen bis zu $650 \mu \mathrm{g} /$ $\mathrm{m}^{3}$ erreicht [93]. NO oxidiert an der Luft rasch zu $\mathrm{NO}_{2}$, das deutlich besser wasserlöslich ist [91]. Die hohe Diffusionsgeschwindigkeit und gute Wasserlöslichkeit der Moleküle führt beim $\mathrm{NO}_{2}$ dazu, dass es rasch von der Schleimhaut wieder absorbiert wird [94]. Das ist jedenfalls für das chemisch ähnliche $\mathrm{SO}_{2}$ nachgewiesen, wo bei Nasenatmung bereits $99 \%$ des Gases in der Nase absorbiert wurde $[95,96]$. In den Bronchien werden vermutlich etwa $10 \%$ des $\mathrm{NO}$ im Exhalat in $\mathrm{NO}_{2}$ umgewandelt [97].

Im Atemtrakt befinden sich also möglicherweise bereits Konzentrationen im Bereich des Grenzwertes für $\mathrm{NO}_{2}$ von $40 \mu \mathrm{g} / \mathrm{m}^{3}$, wenn man davon ausgeht, dass nur $5 \%$ des von der Nase erzeugten NO bereits in den Atemwegen zu NO $\mathrm{N}_{2}$ oxidiert wird [97]. Damit ist es biologisch nicht plausibel, dass aus der Außenluft inhaliertes $\mathrm{NO}_{2}$ in diesen geringen Konzentrationen schädlich sein kann.

Diese Feststellung aus der Physiologie ist gleichzeitig ein weiteres Beispiel dafür, dass aus epidemiologischen Studien keine kausalen Schlüsse gezogen werden können, sofern für die zur Diskussion stehenden Konzentrationen keine experimentellen Befunde vorliegen.

Zusammengefasst hat der geltende Jahresmittelwert von $40 \mu \mathrm{g} / \mathrm{m}^{3}$ für $\mathrm{NO}_{2}$ wissenschaftlich keine ausreichend gesicherte Begründung. Es ist vor dem Hintergrund der hier offengelegten Unsicherheiten von epidemiologischen Studien und dem Mangel an experimentellen Befunden nicht belegbar, dass geringfügige Grenzwertüberschreitungen gesundheitliche Schäden nach sich ziehen. Zudem muss daran erinnert werden, 


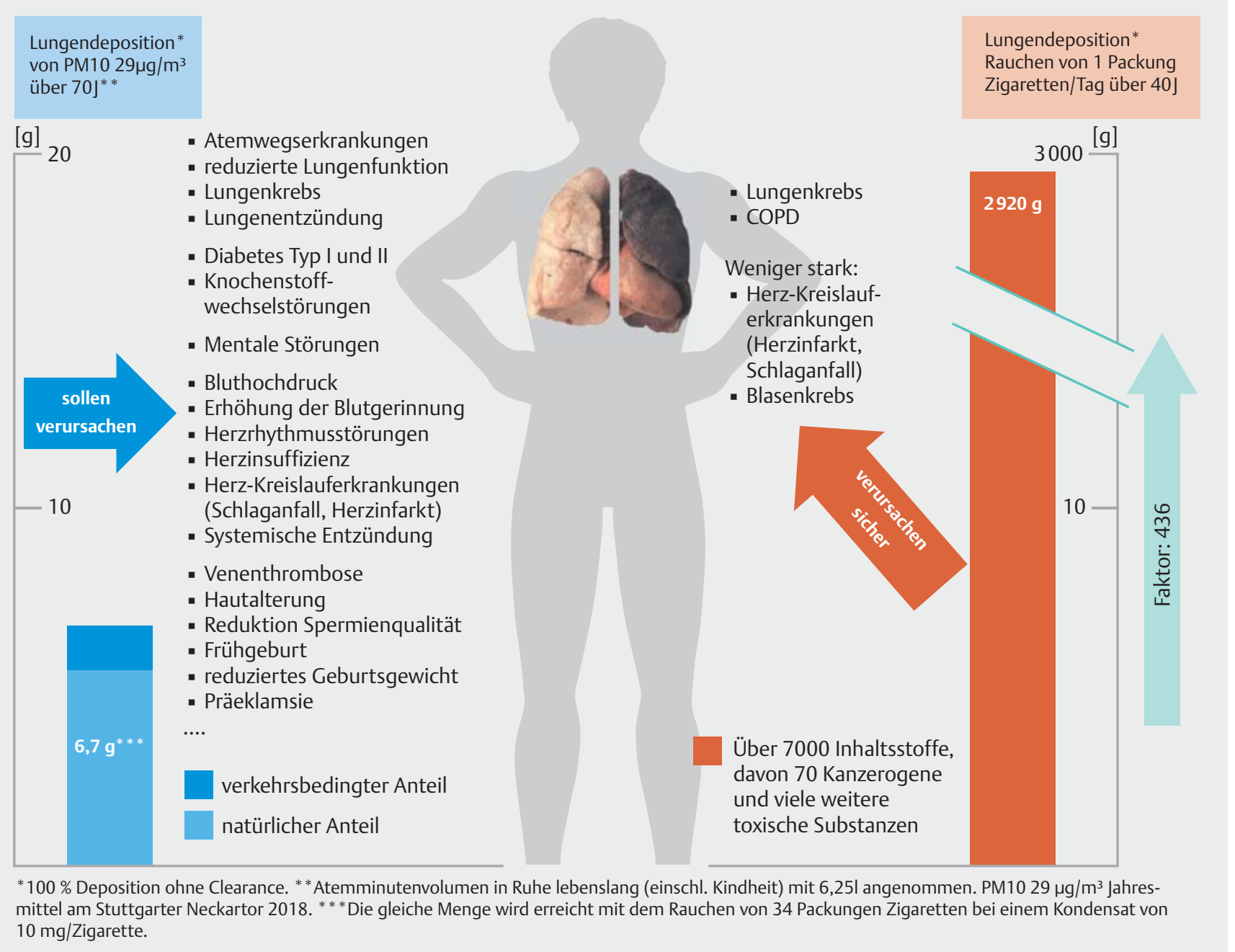

- Abb. 1 Gegenüberstellung der inhalierten Dosen von Feinstaub am Stuttgarter Neckartor (Mittelwert 2018) im Vergleich zum Inhalationsrauchen. Ein Gesunder würde etwa 6,7 g im Laufe seines 70-jährigen Lebens einatmen. Der verkehrsbedingte Anteil am Feinstaub in unmittelbarer Nähe zur Messstation liegt dabei inzwischen bei ca. $4 \mu \mathrm{g} / \mathrm{m}^{3}$, was dann ca. 1,4 g Deposition in $70 \mathrm{~J}$ ausmacht. Nach den epidemiologischen Studien soll diese vergleichsweise geringe Menge eine große Anzahl von Erkrankungen verursachen.

Ein Zigarettenraucher würde bei einer Packung/Tag in $40 \mathrm{~J} 2920 \mathrm{~g}$ Feinstaub inhalieren, also $435 \times$ mehr; bei Bezug auf den verkehrsbedingten Anteil sogar $1537 \times$ mehr. Gesichert ist hier die Verursachung von Lungenkrebs und COPD und weniger stark auch von Herz-Kreislauf-Erkrankungen und Blasenkrebs.

Es gibt zahlreiche Erkrankungen, die mit dem Zigarettenrauch assoziiert sind; es wurden jedoch nur diejenigen erwähnt, für die es eine sehr deutliche Risikoerhöhung und pathophysiologische Hinweise mit Tierversuchen gibt. Im Unterschied zum Feinstaub an befahrenen Straßen ist die nachgewiesene Toxizität des Zigarettenrauchs zudem noch ungleich höher, da durch die Glut und den Tabak viel mehr Reizstoffe und Kanzerogene entstehen.

dass der Grenzwert heute straßennah unterschritten werden muss, sich die Menschen jedoch zu 80 bis $90 \%$ in Gebäuden aufhalten.

\section{Stickoxide als Surrogatparameter für Schadstoffbelastung}

Die Grenzwertfestlegung für Stickoxide wird zusätzlich damit begründet, dass die Schädlichkeit von Stickoxiden selbst nicht entscheidend sei, diese vielmehr zusätzliche indirekte Wirkungen erzeugten und insgesamt als Surrogatparameter für Schadstoffbelastung herangezogen werden können. So schreibt die
Expertise der IHSS und ERS: „Stickoxide sind wichtige Vorläufer von Ozon und tragen zur Bildung von Feinstaub bei.“

Insbesondere die Ozonbildung ist komplex und von mehreren Faktoren abhängig, die sich gegenseitig beeinflussen. Durch den Rückgang der Stickoxidemissionen $\left(\mathrm{NO}_{\mathrm{x}}\right)$ und der volatilen organischen Substanzen im Abgas (VOC) durch verbesserte Motorentechnik und Abgasreinigung, insbesondere durch den weitverbreiteten Katalysatoreinsatz, ist auch die Ozonspitzenkonzentration zurückgegangen, sodass seit Jahren kein Ozonalarm mehr ausgelöst wurde und nur vereinzelt Werte oberhalb von $240 \mu \mathrm{g} / \mathrm{m}^{3}$ gemessen werden [98, 99]. 
Wesentlich ist die Titrationsreaktion im Zusammenspiel mit NO

$\mathrm{NO}+\mathrm{O}_{3}<=>\mathrm{NO}_{2}+\mathrm{O}_{2}$

Es handelt sich bei dieser Darstellung des Leighton-Gleichgewichtes um eine Vereinfachung, da die Rückreaktion nur unter Beisein von UV-Strahlung über den Zwischenschritt der Photolyse von $\mathrm{NO}_{2}$ zu NO plus $\mathrm{O}$ erfolgt.

Die Rückreaktion erfolgt also am Tag durch photolytische Spaltung von $\mathrm{NO}_{2}$. Es kann abhängig von den Randbedingungen sowohl eine Bildung als auch eine Reduzierung von Ozon $\mathrm{O}_{3}$ erfolgen. Der Ozonjahresmittelwert steigt nun v. a. an vielbefahrenen Straßen seit vielen Jahren etwas an, liegt aber deutlich niedriger als im ländlichen Hintergrund [100]. Dies ist mit der über die Jahre abnehmenden $\mathrm{NO}_{\mathrm{x}}$-Emission der Fahrzeuge begründet. Im ländlichen Hintergrund führen großskalige Transportprozesse von $\mathrm{NO}_{\mathrm{x}}$ und Reaktion insbesondere mit biogenen VOCs zur Ozonbildung, die Titration von Ozon mit NO ist dort nicht mehr dominant, Ozon ist folglich im Hintergrund erhöht.

Werte oberhalb der Informationsschwelle von $180 \mu \mathrm{g} / \mathrm{m}^{3}$ werden insbesondere an sonneneinstrahlungsreichen Tagen noch häufiger erreicht, sowohl stadtnah als auch stadtfern. Der photochemische Bildungsmechanismus sowie die oben genannte Titration von $\mathrm{O}_{3}$ mit $\mathrm{NO}$ führen zu einem $\mathrm{O}_{3}$-Tagesgang mit einem Maximum am Nachmittag.

\section{Widersprüche und Ungereimtheiten Schadstoffbelastung durch Fahrzeug-Verkehr}

In der Diskussion um die Schadstoffbelastung der Außenluft steht sehr einseitig der Straßenverkehr, der jedoch nur einen Bruchteil der Feinstaub-Belastung der Außenluft ausmacht. Selbst an Autobahnen und hoch belasteten Straßen im innerstädtischen Bereich sind maximal $6 \mu \mathrm{g} / \mathrm{m}^{3}$ bis $8 \mu \mathrm{g} / \mathrm{m}^{3}$ auf den Verkehr zurückzuführen. Etwa die Hälfte davon ist dem Anteil schwerer Nutzfahrzeuge zuzuschreiben. Die verbleibenden $3 \mu \mathrm{g} / \mathrm{m}^{3}$ bis $4 \mu \mathrm{g} / \mathrm{m}^{3}$ werden durch den PKW-Verkehr verursacht, wobei $50 \%$ dem Abrieb und der Aufwirbelung durch alle PKW-Fahrzeugklassen und lediglich $1 \mu \mathrm{g} / \mathrm{m}^{3}$ bis maximal $2 \mu \mathrm{g} / \mathrm{m}^{3}$ den Abgasen aus den Verbrennungsmotoren der PKWs zuzuordnen [44-47] sind.

Durch das Silvesterfeuerwerk werden bspw. Peaks bis über $600 \mu \mathrm{g} / \mathrm{m}^{3}$ erzeugt. Wirtschaft, Privathaushalte, Elektrizitätsund Fernheizwerke und Landwirtschaft erzeugen zusammen wesentlich höhere Emissionen als der Fahrzeug-Verkehr [101]. Das Umweltbundesamt beziffert den Anteil des Straßenverkehrs an der Feinstaub-Belastung mit 13,8 Prozent [102].

Es muss daher daran erinnert werden, dass Reduktionen der Grenzwerte für eine Feinstaubbelastung durch den FahrzeugVerkehr nur marginale Effekte auf die Gesamt-Exposition der Bevölkerung haben werden, europa- oder gar weltweit betrachtet so gut wie keine $[41,42,47]$.

\section{Implikationen der Messung von Schadstoffen}

Das geltende EU-Recht schreibt vor, an welcher Stelle und in welcher Weise die Schadstoffbelastung gemessen werden soll. Es gestattet insofern Spielraum bei der Aufstellung von Messstationen, dass die Abstände von der Fahrbahn bis zu 10 Metern betragen dürfen. In Deutschland wurde von dieser Möglichkeit meist kein Gebrauch gemacht, was tendenziell zu höheren Messwerten, insbesondere bez. der Stickoxide führt. Das Abstandsquadratgesetz aus der Physik beweist, dass hohe Stickoxidkonzentrationen in unmittelbarer Fahrbahnnähe bereits nach wenigen Metern deutlich reduziert gemessen werden. Andere Vorgaben wie die Gewährleistung eines ausreichenden Vermischens der Emissionen mit der Außenluft oder der geforderte Abstand von Kreuzungsbereichen von $25 \mathrm{~m}$ werden in Deutschland oftmals nicht eingehalten. Zudem ist die Position der Messstation häufiger an Stellen gewählt, an denen höhere Emissionen durch Anfahrvorgänge und/oder Steigungen die Messwerte zusätzlich in die Höhe treiben.

\section{Diesel- und Benzinmotoren sowie Elektrofahrzeuge}

Diesel- und Benzinmotoren unterscheiden sich in ihren Motor-Rohemissionen erheblich. Durch den flächendeckenden Einsatz der Abgasnachbehandlung gleichen sich die relevanten „Tailpipe-out“-Emissionen immer mehr auf niedrigem Niveau an.

Dieselmotoren arbeiten mager mit Luftüberschuss und weisen deshalb im Abgas auch Sauerstoff auf. Beide Motortypen emittieren ca. $73 \% \mathrm{~N}_{2}$ und $10-12 \% \mathrm{H}_{2} 0$. Der Benzinmotor emittiert mehr $\mathrm{CO}_{2}$. Im Zuge der Bemühung um eine Reduktion des $\mathrm{CO}_{2}$-Ausstoßes wurde die Direkteinspritztechnik entwickelt, die ihrerseits allerdings zu einer Erhöhung der Partikelrohemission führte $[103,104]$.

Für die modernste Emissionsstufe EURO6 dtemp $_{\text {EURO6 }}$ dfinal hat die wissenschaftliche Gesellschaft für Kraftfahrzeugtechnik und Motorenbau das Emissionsverhalten dieser Technologie als nahezu immissionsneutral beschrieben [105].

Im Mittel gilt für viele Fahrzeuge dieses Jahrzehntes, dass sich Diesel-PKW durch höhere $\mathrm{NO}_{\mathrm{x}}$-Emissionen auszeichnen (200 bis $800 \mathrm{mg} / \mathrm{km}$ mit Ausreißern und einem $\mathrm{NO}_{2}$-Anteil von bis über 40 Prozent). Fast alle Ottomotoren liegen im Bereich unter $60 \mathrm{mg} / \mathrm{km}$ [106].

Ottomotoren emittieren teilweise bis $6 * 10^{12}$ Partikel/km [107-109]. Dieselfahrzeuge emittieren durch den Einsatz der Partikelfilter mehr als 100-fach weniger, womit sie im Bereich der unbelasteten Außenluft liegen. Mit der bereits laufenden Einführung der EURO6 dtemp $_{\text {-Gesetzgebung werden auch alle }}$ Ottomotoren spätestens ab 1. September 2019 einen Partikelfilter erhalten.

Insgesamt weisen Dieselfahrzeuge weiterhin einen höheren Wirkungsgrad auf als Ottomotoren, weswegen sie bei gleicher Leistung rund 15 Prozent weniger $\mathrm{CO}_{2}$ produzieren. Das kommt den angestrebten $\mathrm{CO}_{2}$-Zielen entgegen.

Elektrofahrzeuge erzeugen keine direkten Emissionen durch Verbrennungsprozesse, jedoch ebenfalls durch fahrzeugseitige Effekte. Zudem unterliegen auch diese Fahrzeuge einer Energiebilanzierung. Die aktuell noch ungelösten Probleme der 
Elektromobilität (extrem hoher Strombedarf, Bau und Entsorgung von Batterien) sprechen nicht dafür, Grenzwerte zum Vehikel der schleichenden Elimination von Verbrennungsmotoren zu machen. Angemessener erscheint vor dem Hintergrund der weltweiten Notwendigkeit der $\mathrm{CO}_{2}$-Reduktion eine intensive Bemühung um neue Antriebs-Technologien.

\section{Unterschiedliche Grenzwerte für die Außenluft und Arbeitsplätze}

Die geltenden Grenzwerte für eine $\mathrm{NO}_{2}$-Exposition liegen in Deutschland für die Außenluft bei $40 \mu \mathrm{g} / \mathrm{m}^{3}$, am Arbeitsplatz bei $950 \mu \mathrm{g} / \mathrm{m}^{3}$. Für Büro- und Wohnräume gibt es nur einen Richtwert von $60 \mu \mathrm{g} / \mathrm{m}^{3}$, wobei das Umweltbundesamt (UBA) in einem Protokoll vom Dez. 2018 inzwischen [110] sogar davon spricht, dass erst ab $500 \mu \mathrm{g} / \mathrm{m}^{3}$ belastbare Daten für Kurzzeiteffekte bei Personen mit leichtem Asthma vorliegen. Diese Unterschiede werden damit begründet, dass gegenüber der Außenluft eine dauerhafte Exposition bestehe, während sie andernorts nur temporär sei.

Tatsächlich macht diese Unterscheidung keinen Sinn. Eine grenzwertnahe Exposition von $8 \mathrm{~h}$ pro Tag in Innenräumen bzw. $40 \mathrm{~h}$ in der Woche dürfte wesentlich intensiver sein als eine Exposition von 2-4h pro Tag, an denen sich ein Mensch im Freien aufhält, denn dort wechselt er seine Position i.d.R. viel häufiger und bleibt selten ständig im Grenzwertbereich. Das UBA erwähnt auf seiner Homepage, dass sich Menschen zu $90 \%$ in Innenräumen aufhalten [111].

Feinstaubkonzentrationen, egal zu welcher Korngröße, sind aufgrund der natürlich bedingten Grundbelastung und witterungsabhängigen Schwankungen als Immissionsgrenzwerte für die Außenluft keine geeignete Messgröße. Sinnvoll ist allein die emissionsseitige Begrenzung der anthropogenen Quellen.

\section{Bisherige Effekte der Umweltpolitik}

Schließlich ist es notwendig, auf das bisher Erreichte in der Umweltpolitik bzw. die Reduktion der Schadstoffe hinzuweisen.

Ausweislich der Angaben des Umweltbundesamtes konnte die Belastung durch Feinstaub (PM10) von 1990 bis 2016 von 328 auf 203 Tausend Tonnen zurückgeführt werden [112]. Ebenso wurde die Belastung durch Stickoxide in diesem Zeitraum von 2892 auf 1217 Tausend Tonnen reduziert, also mehr als halbiert [113]. Darüber hinaus reduzierte sich auch kontinuierlich der relative Anteil des Verkehrs an der Schadstoffbelastung.

Das Umweltbundesamt schreibt: „Während zu Beginn der 1990er-Jahre im Jahresmittel großräumig Werte um 50 Mikrogramm pro Kubikmeter $\left(\mu \mathrm{g} / \mathrm{m}^{3}\right)$ gemessen wurden, treten heute PM10-Jahresmittelwerte zwischen 15 und $20 \mu \mathrm{g} / \mathrm{m}^{3}$ auf." Grenzwertüberschreitungen sind heute eine extreme Ausnahme [102].

\section{Sommer-Winter-Vergleich}

Ein wesentlicher potenzieller Störfaktor sind die jahreszeitlichen Änderungen der Wetterbedingungen. So sind in vielen Regionen, bedingt durch häufige Inversionswetterlagen, die Feinstaub- und $\mathrm{NO}_{2}$-Konzentrationen im Winter doppelt so hoch wie im Sommer. Gleichzeitig ist die Mortalität im Winter deutlich größer als im Sommer. Dieser deutliche Zusammenhang könnte nun dadurch erklärt werden, dass die schlechte Luftqualität im Winter die Mortalität erhöht, aber auch damit, dass die gehäuften viralen und bakteriellen Infektionen im Winter oder einfach die Kälte die Mortalität erhöhen. Während austauscharmer Wetterlagen sind nicht nur die inhalierbaren Schadstoffe erhöht, sondern auch die infektiösen Aerosole [114]. Erst seit einigen Jahren weiß man, dass bereits durch die Ausatmung, also auch ohne Husten, der Mensch immer Aerosole um $1 \mu \mathrm{m}$ erzeugt [115]. Diese können infektiöse Erreger, insbesondere Viren, enthalten $[116,117]$. Nach Wissen der Autoren wurde das Problem bisher in keiner epidemiologischen Studie berücksichtigt oder diskutiert.

Vor diesem Hintergrund von Unsicherheiten sind zum jetzigen Zeitpunkt rigorose Maßnahmen zur Reduktion von Grenzwertüberschreitungen nicht verhältnismäßig. Stattdessen sollte kontinuierlich durch technologische Fortschritte an der Reduktion der emittierten Schadstoffbelastung gearbeitet werden.

\section{Entgegnungen}

\section{Rauchen}

Die Initiative deutscher Lungenärzte weist darauf hin, dass die Exposition durch Rauchen eine ungleich höhere Feinstaubbelastung impliziert und aufgrund dessen die postulierten Gesundheitsrisiken der Feinstaubexposition durch Verkehr erheblich zu relativieren sind.

Der „Großversuch“ Rauchen falsifiziert die Hypothese der Schädlichkeit von Feinstaub in der legislativen Grenzwertdosis:

Konkret gerechnet, ergibt sich folgendes Bild: Der Mensch atmet in Ruhe ca. $9 \mathrm{~m}^{3} /$ Tag oder ca. $260000 \mathrm{~m}^{3}$ in seinem Leben ein (bei angenommenen 80 Jahren, ohne Berücksichtigung des reduzierten Atemvolumens in der Kindheit und im Schlaf). Bei einer lebenslangen Inhalation von $50 \mu \mathrm{g} / \mathrm{m}^{3}$ Feinstaub (hier wurde eine Deposition von $100 \%$ angenommen; obwohl die Deposition bei Spontanatmung unter $50 \%$ liegt [118]) ergibt sich im ungünstigsten Falle eine lebenslange Belastung von ca. $13 \mathrm{~g}$ PM10. Zum Vergleich: Pro Zigarette werden aktuell max. $10 \mathrm{mg}$ Tabakverbrennungsprodukte in der Lunge deponiert, da im Gegensatz zu früher die Kondensatdosis durch EU-Verordnung reduziert wurde. Bei einer angenommenen Deposition von $100 \%$ der Zigarettenrauchpartikel (realistische Deposition beim Raucher ca. 80\% [119-121]) erreicht der Raucher die gleiche retinierte Dosis von $13 \mathrm{~g}$ bereits nach ca. 1300 Zigaretten oder nach ca. 65 Packungen (gut 2 Monate bei 1 Packung/ Tag).

Hinzu kommt noch erschwerend, dass der Zigarettenrauch ungleich toxischer ist als der Feinstaub [122-124]. Einen Vergleich der inhalierten Dosen aus dem Zigarettenrauch und der Jahresmittelkonzentration PM10 am Stuttgarter Neckartor zeigt $>$ Abb. 1.

Die ISEE- und ERS-Expertise hatte erwidert, dass im Falle des Feinstaubs keine lineare Dosis-Wirkungs-Beziehung hinsichtlich kardiovaskulärer Erkrankungen besteht.

Dieser Einwand eines nicht-linearen Zusammenhangs von Exposition und Effekt trifft in dieser Form nicht zu. Mit zuneh- 
mender Rauchdosis steigt das relative Risiko für eine koronare Herzerkrankung $[125,126]$ oder Mortalität $[123,127]$ deutlich an. Ebenso besteht eine klare Dosis-Wirkungs-Beziehung zwischen Rauchen und Lungenkrebs [37,128-31]. In gleicher Weise gilt diese Assoziation auch für die COPD [131, 132]. Zu beachten ist dabei, dass bei der Risikoschätzung von Tabakrauchen eine kumulative Dosis (Packungsjahre) zugrunde gelegt wird, bei Feinstaub hingegen wird lediglich eine Konzentration, extrapoliert auf den Untersuchungszeitpunkt, als Expositionsmaß genommen.

Darüber hinaus weist die Expertise der ISEE und ERS auf die Unterschiede in der Dauer der Exposition hin (kurze hohe Exposition beim Rauchen, geringere, aber andauernde Exposition durch Umgebungsluft).

Bislang wurde in epidemiologischen Studien noch nicht ausreichend detailliert untersucht, ob eine kurze hohe Exposition weniger schädlich ist als eine andauernde niedrige, wobei die hohe Innenraumbelastung in den Rauchpausen noch hinzukommt. Die durch Rauchen deponierte Menge Feinstaub verweilt in der Lunge, was auch im Gewebe und in den Makrophagen unmittelbar sichtbar ist. Die Konstruktion einer andauernden Umweltexposition ist ebenfalls falsch. Die Exposition und Dauer der Belastung wechseln, weil alle Menschen regelmäßig ihre Position ändern, sich im Laufe des Tages am Arbeitsplatz und zu Hause aufhalten, unterwegs sind, Freizeitaktivitäten ausüben und in den Urlaub fahren. Insofern ist es wichtig, die Exposition gegenüber Feinstaub mit personengetragenen Messgeräten zu validieren.

Dass Rauchen in erster Linie erwachsene Menschen betrifft, ist ebenfalls nicht richtig. Bspw. werden Kinder rauchender Mütter mit einem geringeren Gewicht geboren. Dies ist per se jedoch ebenso wenig ein Argument für die geltenden Grenzwerte des Feinstaubs wie der Hinweis darauf, dass das Rauchen ja auch eingestellt werden kann, da es hier um die Frage der Verhältnismäßigkeit - der relativen Schädlichkeit von Rauchen und Feinstaubexposition - geht. Rauchen stellt gewissermaßen einen toxikologischen Expositionsversuch dar, der sonst wegen der ethischen Limitation nicht möglich wäre.

\section{Vergiftungsmuster}

Die ISEE- und ERS-Expertise verweist darauf, dass es ein typisches Vergiftungsmuster der Schadstoffe sehr wohl in Form eines oxidativen Stresses sowie entzündlicher Reaktionen gibt, das für unterschiedlichste Folgen, von der Demenz bis zum Diabetes, verantwortlich gemacht werden kann.

Diese Annahme ist in der Theorie zulässig. Für den diskutierten Niedrigdosisbereich von Schadstoffen ist die Annahme eines dadurch ausgelösten dramatischen oxidativen Stresses sehr wenig plausibel, zumal die Evolution entsprechende Abwehrmechanismen entwickelt hat.

Die Vielfalt der gefundenen Assoziationen, sämtlich in schwacher Ausprägung, unterstützt jedoch nicht zusätzlich eine Bedeutung der Feinstaub-Expositionen für die Gesundheit, sondern stellt sie eher infrage. Nach dem Wissen der Autoren fehlen geeignete Tierversuche im Niedrigdosisbereich.

Häufig wird erwähnt, dass Feinstaub Mediatoren der Entzündung in Zellkulturen oder im Blut induzieren würde. Diese wür- de dann zu Krankheiten führen. Solche Mediatoren sind jedoch eine unspezifische Abwehrreaktion, die man praktisch auf jeden Fremdstoff oder auch schon auf körperliche Belastung messen kann [133]. Ein pathophysiologisches Konzept für die zahlreichen Krankheitsbilder, deren Entstehung dem Feinstaub angelastet wird, ist experimentell unter den diskutierten Konzentrationen nicht belegt.

Schlüssiger wären Tiermodelle. Hier hätte man erwartet, dass man bei dem großen Forschungsaufwand, der für Feinstaub getrieben wird, ausreichend belastbare Ergebnisse in Bezug auf die zahlreichen in den epidemiologischen Studien gefundenen Erkrankungen finden würde. Dies ist jedoch nicht der Fall. Zahlreiche experimentelle Untersuchungen gibt es bspw. für die Toxizität von Zigarettenrauch [123, 134].

Regelhaft wird auf Veröffentlichungen hingewiesen, in denen transgen veränderte Mäuse in Verbindung mit cholesterinreicher Kost mehr Atherosklerose entwickeln, wenn sie etwa zehnfach aufkonzentrierten Feinstaub aus Innenstädten inhalieren im Vergleich zu einer Kontrollgruppe [135]. Dies steht im Widerspruch zu den letzten großen epidemiologischen Metaanalysen an über 300000 Personen, in denen sich kein Einfluss des Feinstaubs mehr auf die kardiorespiratorische Mortalität zeigte [5].

Diese Ergebnisse weisen auf einen systematischen Fehler hin, den man häufig in der Literatur zum Feinstaub oder $\mathrm{NO}_{\mathrm{x}}$ findet. Obwohl in der Literatur andere Erklärungsmodelle existieren, werden sie nicht zitiert.

Das Modell mit den transgenen Mäusen, die auf cholesterinreiche Kost verstärkt eine Arteriosklerose entwickeln, wird in verschiedenen Bereichen angewendet, auch zur Stressforschung. Hier findet sich allein durch Anwendung von unregelmäßigem Stress im Tierlabor eine ähnliche und sogar beschleunigte Zunahme der Arteriosklerose [136]. Ähnliches wurde auch bei genetisch unveränderten Kaninchen gefunden, und zwar bereits auf milde Stressreaktionen [137].

Es ist daher plausibel, dass in dem konzentrierten Feinstaub aus den Innenstädten viele Geruchsstoffe von Katzen, Hunden, aber auch Autoabgasen enthalten sind, die bei den Versuchstieren eine natürliche Fluchtreaktion auslösen, die aber im Käfig frustran bleiben muss. Das erzeugt natürlich maximalen Stress.

Für diese Hypothese spricht, dass allein die Gabe von Geruchsstoffen von Raubtieren bereits bei nicht genetisch veränderten Mäusen Veränderungen im Gehirn auslösen kann [138].

Diese Modelle wären also nur valide, wenn sie eine weitere Kontrollgruppe mit vergleichbarer Belastung mit Stressoren enthalten würden.

\section{Tote durch Feinstaub und Stickoxide}

Die ISEE- und ERS-Expertise weist das Argument zurück, dass Effekte der Schadstoff-Belastung in der Luft für Kliniker nicht wahrnehmbar sind. Sie begründet diese Fragestellung damit, dass Zusammenhänge von Erkrankung und Exposition immer nur epidemiologisch und toxikologisch belegt werden können.

Dies trifft grundsätzlich zu. Allerdings sind die Folgen des Rauchens klinisch sichtbar mit einer „schwarzen“ Lunge und ebenso offensichtlich bei Lungenärzten in Form von COPD und Lungenkrebs. V.a. (keineswegs ausschließlich) sind Plattenepi- 
thelkarzinome und kleinzellige Lungenkarzinome typische Rauchererkrankungen. Ein auch nur annähernd ähnlicher Zusammenhang zu Feinstaub und Stickoxiden ist für Lungenärzte nicht erfahrbar; dies weist auf das drastische Missverhältnis der Bedeutung beider Einflussgrößen für die Verursachung tödlich verlaufender oder lebenszeitverkürzender Erkrankungen hin.

Das prinzipielle Problem eines Zusammenhangs zwischen Exposition und Erkrankung wird in der Arbeitsmedizin bei Berufserkrankungen aufwendig ermittelt, wo zwischen Exposition und klinisch nachgewiesener Berufserkrankung (z. B. Silikose) ein Zusammenhang mit hoher Wahrscheinlichkeit für eine Entschädigung dargestellt werden muss.

In der UBA-Publikation werden etwa 6000 Sterbefälle dem $\mathrm{NO}_{2}$ angelastet [139]. Es wurde nachgewiesen, dass die Berechnung der Todesfälle aus den epidemiologischen Untersuchungen über das sog. attributable Risiko nicht nur wegen der fehlenden Kausalität falsch, sondern - wenn man das Risiko als gegeben annehmen würde - auch mathematisch unrichtig ist [140]. Richtig wäre die Angabe von reduzierter Lebenszeit (YLL, Years of Life Lost). Bei der in der UBA-Publikation angegebenen YLL/100 00 Einwohner von 87,96 wären das dann ca. 8 Std./Einwohner Lebenszeitverkürzung. Dagegen verlieren die Deutschen durch ungesunden Lebensstil mehrere Lebensjahre, und zwar Männer bis zu 17 Jahre [38].

\section{Kanzerogenität des Feinstaubs}

Die chemische Zusammensetzung des Feinstaubs ist lokal sehr unterschiedlich. Das kann natürliche (z. B. Aerosole, Salze in Meeresnähe, mineralische Anteile in Wüstengebieten, Vulkanasche u.a.) oder anthropogene Ursachen (z. B. Brandrodung, Industrieabgase, Heizungsanlagen, Verkehr, Abrieb in U-Bahnstationen u.a.) haben. Als Feinstaub werden auch biogene Anteile (z. B. Pollen) gemessen.

Auch aus diesem Grund ist es sehr fragwürdig, allgemein von einer karzinogenen Wirkung des Feinstaubs zu sprechen; ein solcher Effekt müsste einer oder mehreren Substanzgruppen zuzuordnen, tierexperimentell als karzinogen nachgewiesen und epidemiologisch reproduzierbar zu zeigen sein. Diese Beweiskette ist jedoch bislang nicht vorgelegt worden.

\section{Unterschiede in den Grenzwerten EU versus USA}

Während in der EU der Grenzwert für $\mathrm{NO}_{2} 40 \mu \mathrm{g} / \mathrm{m}^{3}$ beträgt, wurde in den USA ein Grenzwert von ca. $100 \mu \mathrm{g} / \mathrm{m}^{3}$ festgelegt. Auch die in Kalifornien und weiteren 16 Bundesstaaten geltenden strengeren Stickoxid-Grenzwerte von $57 \mu \mathrm{g} / \mathrm{m}^{3}$ würden Fahrverbote in Deutschland infolge von Grenzwertüberschreitungen weitgehend ausschließen.

Die ISEE- und ERS-Expertise argumentiert: Dafür hätten die USA deutlich schärfere Grenzwerte für die Emission von Fahrzeugen. Dieser Einwand ist nicht stichhaltig, da die letztlich eingeatmete Umgebungsluft entscheidend für eine Exposition ist, nicht der Emissionsgrenzwert. Es bleibt daher dabei, dass in den USA ein zweieinhalbfach höherer Grenzwert akzeptiert wird.

Schließlich weist die Expertise auf den nur halb so großen Grenzwert für Feinstaub (PM2,5) hin, der in den USA gilt $\left(12 \mu \mathrm{g} / \mathrm{m}^{3}\right.$ versus $\left.25 \mu \mathrm{g} / \mathrm{m}^{3}\right)$.
Diese Tatsache ist jedenfalls nicht vereinbar mit der häufig geäußerten Position, $\mathrm{NO}_{2}$ sei nicht selbst schädlich, sondern ein Korrelat für die Feinstaubbelastung.

In der Schweiz liegt ein niedriger $\mathrm{NO}_{2}$-Grenzwert von $30 \mu \mathrm{g} /$ $\mathrm{m}^{3}$ vor. Dieser ist jedoch v. a. aus Biodiversitätsgründen zum Schutze der Artenvielfalt auf dem Lande niedrig gewählt. Auch in Deutschland wird dieser Wert im Hintergrundmessbereich typischerweise schon heute eingehalten.

\section{Ethische Überlegungen}

Dem Staat kommt gegenüber seinen Bürgern eine Fürsorgepflicht zu. Interventionen, die dieses Ziel verfolgen, müssen 1) wirksam und 2) verhältnismäßig sein. Keinesfalls gibt es eine absolute Fürsorgepflicht des Staates, denn der Staat kann immer nur eine Risikominimierung, niemals aber eine Risikoelimination, leisten. Dies ergibt sich schon daraus, dass eine Risikoelimination prinzipiell unmöglich ist. Die Fürsorgepflicht vollzieht sich somit i.d. R. im Rahmen einer Güterabwägung.

Die Wirksamkeit für eine gesundheitliche Risikoreduktion von rigorosen Maßnahmen zur Verhinderung von Grenzwertüberschreitungen sowie der weiteren Reduktionen von Grenzwerten muss angezweifelt werden. Für $\mathrm{NO}_{2}$ liegt keine überzeugende Evidenz für gesundheitliche Risiken in aktuell erzielten Konzentrationen vor, wie insbesondere die aktuelle Meta-Analyse in ESCAPE gezeigt hat [11]. Adäquate Tierversuche, insbesondere im Niedrigdosisbereich, fehlen.

Die Steuerungsmöglichkeiten der Feinstaubimmissionen sind auf den geringeren anthropogenen Anteil begrenzt.

Vor dem Hintergrund dieser Unsicherheiten erscheinen die aktuell durchgeführten oder anstehenden Fahrverbote als völlig unverhältnismäßig. Die Tatsache, dass Lifestyle-Risikofaktoren wie Rauchen, Übergewicht, übermäßiger Konsum von Alkohol und rotem Fleisch zusammen mit einer Einbuße an Lebenszeit von 17 Jahren für Männer und 14 Jahren für Frauen assoziiert sind [38], zeigt deutlich, wo die wesentlichen individuell beeinflussbaren Gesundheitsrisiken liegen und wo die Prävention mit großem Erfolgspotenzial erfolgen sollte.

Schließlich sind auch die sozialen und anderen Folgen unangemessener und unverhältnismäßiger Interventionen zu beachten. Dazu gehören neben der finanziellen Belastung größerer Bevölkerungsgruppen auch die negativen ökologischen Schäden einer vorzeitigen Abwrackung von älteren Dieselfahrzeugen und unnötiger Herstellung von Neuwagen.

\section{Danksagung}

Wir danken Herrn Prof. Dr. med. univ. Horst Olschewski (Graz) und Herrn Prof. Dr. med. Matthias Griese (München) herzlich für ihre kritische Durchsicht des Manuskriptes. 


\section{Interessenkonflikt}

D. Köhler, M. Hetzel, M. Klingner, S. Ewig, G. Becher, H. Lindemann, T. Voshaar, und U. Costabel geben an, dass kein Interessenkonflikt besteht.

T. Koch: Das Institut für Kolbenmaschinen am KIT finanziert sich neben einer Basisfinanzierung zu einem überwiegenden Maße durch öffentliche Drittmittel von Ministerien, Stiftungen, Fördergesellschaften oder der Forschungsvereinigung FVV, typischerweise mit Fördermitteln der AIF oder Cornet. Ein deutlich kleinerer Anteil wird durch Drittmittel aus einer weitgefächerten Industrielandschaft (Energietechnik, Zulieferindustrie, Messtechnik, Fertigungstechnik, Fahrzeug- und Motorenindustrie) durch Forschungsaktivitäten beigesteuert. Ein Interessenkonflikt oder gar eine Beeinflussung hinsichtlich der Beiträge in dieser Publikation liegen nicht vor.

\section{Literatur}

[1] Nicht auf der Webseite der ERS/ISEE verfügbar. z. B. unter: https:// www.duh.de/fileadmin/user_upload/download/Pressemitteilungen/Verkehr/Die_Rolle_der_Luftschadstoffe_f\%C3\%BCr_die_Gesundheit_-_Expertise_der_ISEE_ERS.pdf. 2019

[2] https://cfpub.epa.gov/ncea/risk/recordisplay.cfm?deid=216546. 2009

[3] https://www.epa.gov/isa/integrated-science-assessment-isa-nitrogen-dioxide-health-criteria. 2016

[4] https://cordis.europa.eu/project/rcn/88859/factsheet/en. 2017

[5] Wang M, Beelen R, Stafoggia M et al. Long-term exposure to elemental constituents of particulate matter and cardiovascular mortality in 19 European cohorts: results from the ESCAPE and TRANSPHORM projects. Environ Int 2014; 66: 97 - 106

[6] Beelen R, Stafoggia M, Raaschou-Nielsen O et al. Long-term exposure to air pollution and cardiovascular mortality: an analysis of 22 European cohorts. Epidemiology 2014; 25: 368 - 378

[7] Brunekreef B, Beelen R, Hoek G et al. Effects of long-term exposure to traffic-related air pollution on respiratory and cardiovascular mortality in the Netherlands: the NLCS-AIR study. Res Rep Health Eff Inst 2009; 139: 5-71

[8] Carey IM, Atkinson RW, Kent AJ et al. Mortality associations with long-term exposure to outdoor air pollution in a national English cohort. Am J Respir Crit Care Med 2013; 187: 1226-1233

[9] Cesaroni G, Badaloni C, Gariazzo C et al. Long-term exposure to urban air pollution and mortality in a cohort of more than a million adults in Rome. Environ Health Perspect 2013; 121: 324 - 331

[10] Turner MC, Jerrett M, Pope CA3rd et al. Long-Term Ozone Exposure and Mortality in a Large Prospective Study. Am J Respir Crit Care Med 2016; 193: $1134-1142$

[11] Beelen R, Raaschou-Nielsen O, Stafoggia M et al. Effects of longterm exposure to air pollution on natural-cause mortality: an analysis of 22 European cohorts within the multicentre ESCAPE project. Lancet 2014; 383: $785-795$

[12] Beelen R, Hoek G, Raaschou-Nielsen O et al. Natural-cause mortality and long-term exposure to particle components: an analysis of 19 European cohorts within the multi-center ESCAPE project. Environ Health Perspect 2015; 123: 525-533

[13] https://www.umweltbundesamt.de/daten/luft/luftschadstoff-emissionen-in-deutschland/schwefeldioxid-emissionen\#textpart-1. 2018

[14] Lederer DJ, Bell SC, Branson RD et al. Control of Confounding and Reporting of Results in Causal Inference Studies. Guidance for Authors from Editors of Respiratory, Sleep, and Critical Care Journals. Ann Am Thorac Soc 2019; 16: 22 - 28
[15] Andersen ZJ, Stafoggia M, Weinmayr G et al. Long-Term Exposure to Ambient Air Pollution and Incidence of Postmenopausal Breast Cancer in 15 European Cohorts within the ESCAPE Project. Environ Health Perspect 2017; 125: 107005

[16] Faridi S, Shamsipour M, Krzyzanowski M et al. Long-term trends and health impact of $\mathrm{PM}(2.5)$ and $\mathrm{O}(3)$ in Tehran, Iran, 2006-2015. Environ Int 2018; 114: 37 -49

[17] Lipfert FW. A critical review of the ESCAPE project for estimating long-term health effects of air pollution. Environ Int 2017; 99: 87 96

[18] Lipfert FW. Letter to the Editor Re: Enstrom JE. Fine particulate and total mortality in Cancer Prevention Study cohort reanalysis. DoseResponse 2017; 15: 1 - 12

[19] Stafoggia M, Cesaroni G, Peters A et al. Long-term exposure to ambient air pollution and incidence of cerebrovascular events: results from 11 European cohorts within the ESCAPE project. Environ Health Perspect 2014; 122: $919-925$

[20] Kabat GC. Hyping Health Risks: Environmental Hazards in Daily Life and the Science of Epidemiology. New York: Columbia University Press Irvington; 2008

[21] Krieger N. Epidemiology and the web of causation: has anyone seen the spider? Soc Sci Med 1994; 39: 887 - 903

[22] Taubes G. Epidemiology faces its limits. Science 1995; 269: 164 169

[23] Singh S, Loke YK, Furberg CD. Inhaled anticholinergics and risk of major adverse cardiovascular events in patients with chronic obstructive pulmonary disease: a systematic review and meta-analysis. JAMA 2008; 300: 1439-1450

[24] Tashkin DP, Celli B, Senn S et al. UPLIFT Study Investigators. A 4-year trial of tiotropium in chronic obstructive pulmonary disease. N Engl Med 2008; 359: 1543-1554

[25] Wise RA, Anzueto A, Cotton D et al. TIOSPIR Investigators. Tiotropium Respimat inhaler and the risk of death in COPD. N Engl J Med 2013; 369: 1491 - 1501

[26] Gupta RP, Mukherjee M, Sheikh A et al. Persistent variations in national asthma mortality, hospital admissions and prevalence by socioeconomic status and region in England. Thorax 2018; 73: 706 712

[27] Hovanec J, Siemiatycki ], Conway DI et al. Lung cancer and socioeconomic status in a pooled analysis of case-control studies. PLoS One 2018; 13: e0192999

[28] Nordahl H. Social inequality in chronic disease outcomes. Dan Med 2014: 61: B4943

[29] Ohlmeier C, Langner I, Hillebrand K et al. Mortality in the German Pharmacoepidemiological Research Database (GePaRD) compared to national data in Germany: results from a validation study. BMC Public Health 2015; 15: 570

[30] Ponce NA, Hoggatt KJ, Wilhelm M et al. Preterm birth: the interaction of traffic-related air pollution with economic hardship in Los Angeles neighborhoods. Am J Epidemiol 2005; 162: 140 - 148

[31] Ratjen I, Schafmayer C, Enderle J et al. Health-related quality of life in long-term survivors of colorectal cancer and its association with all-cause mortality: a German cohort study. BMC Cancer 2018; 18 : 1156

[32] Smith SG, Jackson SE, Kobayashi LC et al. Social isolation, health literacy, and mortality risk: Findings from the English Longitudinal Study of Ageing. Health Psychol 2018; 37: 160-169

[33] Vestbo J, Anderson JA, Calverley PM et al. Adherence to inhaled therapy, mortality and hospital admission in COPD. Thorax 2009; 64 : $939-943$

[34] Ding P, VanderWeele T], Robins JM. Instrumental variables as bias amplifiers with general outcome and confounding. Biometrika 2017; 104: 291 - 302 
[35] VanderWeele T], Hernán MA, Robins JM. Causal directed acyclic graphs and the direction of unmeasured confounding bias. Epidemiology 2008; 19: 720 - 728

[36] VanderWeele T], Shpitser I. On the definition of a confounder. Ann Stat 2013; 41: $196-220$

[37] Pesch B, Kendzia B, Gustavsson P et al. Cigarette smoking and lung cancer-relative risk estimates for the major histological types from a pooled analysis of case-control studies. Int J Cancer 2012; 131: $1210-1219$

[38] Li K, Hüsing A, Kaaks R. Lifestyle risk factors and residual life expectancy at age 40: a German cohort study. BMC Med 2014; 12: 59

[39] https://www.epa.gov/pm-pollution/2006-national-ambient-airquality-standards-naaqs-particulate-matter-pm25. 2006

[40] https://www.epa.gov/naaqs/particulate-matter-pm-air-qualitystandards. 2015

[41] van Donkelaar A, Martin RV, Brauer M et al. Global Estimates of Ambient Fine Particulate Matter Concentrations from Satellite-Based Aerosol Optical Depth: Development and Application. Environ Health Perspect 2010; 118: $847-855$

[42] Philip S, Martin RV, van Donkelaar A et al. Global chemical composition of ambient fine particulate matter for exposure assessment. Environ Sci Technol 2014; 48: 13060 - 13068

[43] Klingner M, Sähn E. Feinstaub, was man darüber wissen sollte. Kurzbericht im Auftrag des VDA. https://cloud.ivi.fraunhofer.de/s/ mB93zHpz7Lym7Eo. 2006

[44] Klingner M, Sähn E, Anke K et al. Reduktionspotenziale verkehrsbeschränkender Maßnahmen in Bezug zu meteorologisch bedingten Schwankungen der PM10- und NOx-Immissionen. In: Gefahrstoffe Reinhaltung der Luft. Düsseldorf: Springer-VDI-Verlag; 2006: 326 334. https://cloud.ivi.fraunhofer.de/s/mB93zHpz7Lym7Eo

[45] Klingner M, Sähn E. Prediction of PM10 concentration on the basis of high resolution weather forecasting. Meteorologische Zeitschrift 2008; 17: $263-272$. (https://cloud.ivi.fraunhofer.de/s/ mB93zHpz7Lym7Eo)

[46] Rost J, Holst T, Sähn E et al. Variability of PM10 concentrations dependent on meteorological conditions. International Journal of Environment and Pollution (IJEP) 2009; 36, No.1/2/3: 3-18 (3), Interscience Publishers, Geneve, Switzerland, (https://cloud.ivi.fraunhofer.de/s/mB93zHpz7Lym7Eo)

[47] Klingner M, Sähn E. Auswirkungen ordnungsrechtlicher Verkehrsmaßnahmen auf die lokale Feinstaubbelastung unter Berücksichtigung meteorologischer Einflüsse. Im Auftrag des Bundesministeriums für Verkehr, Bau- und Wohnungswesen, 2006. https://cloud.ivi. fraunhofer.de/s/mB93zHpz7Lym7Eo

[48] Hesterberg TW, Long CM, Bunn WB et al. Health effects research and regulation of diesel exhaust: an historical overview focused on lung cancer risk. Inhal Toxicol 2012; 24 (Suppl. 01): 1-45

[49] Mauderly JL. Toxicological and epidemiological evidence for health risks from inhaled engine emissions. Environ Health Perspect 1994; 102 (Suppl. 04): 165-171

[50] Mayer A, Wyser M, Czerwinski C et al. Erfahrungen mit PartikelfilterNachrüstungen bei Baumaschinen in der Schweiz. FAD-Konferenz 2003

[51] Mayer A, Matter U, Scheidegger G et al. Particulate Traps for RetroFitting Construction Site Engines VERT: Final Measurements and Implementation. 1999. https://doi.org/10.4271/1999-01-0116, SAE

[52] Behera D, Jindal SK. Respiratory symptoms in Indian women using domestic cooking fuels. Chest 1991; 100: 385-388

[53] Herbarth O, Fritz G, Krumbiegel P et al. Effect of sulfur dioxide and particulate pollutants on bronchitis in children - a risk analysis. Environ Toxicol 2001; 16: 269-276
[54] Pérez-Padilla R, Regalado J, Vedal S et al. Exposure to biomass smoke and chronic airway disease in Mexican women. A case-control study. Am J Respir Crit Care Med 1996; 154: 701 - 706

[55] Berzon R, Utkin WW, Marga J et al. [Comparative investigations to the epidemiology of chronic bronchitis in Erfurt and Riga (author's transl)]. Z Erkr Atmungsorgane 1980; 155: 341 - 351 Deutsch.

[56] Peters A, Breitner S, Cyrys J et al. The influence of improved air quality on mortality risks in Erfurt, Germany. Res Rep Health Eff Inst 2009; 137: 5-77

[57] Nieminen P, Panychev D, Lyalyushkin S et al. Environmental exposure as an independent risk factor of chronic bronchitis in northwest Russia. Int J Circumpolar Health 2013. doi:10.3402/ijch. v72iO. 19742

[58] Nowak D, Heinrich J, Jörres R et al. Prevalence of respiratory symptoms, bronchial hyperresponsiveness and atopy among adults: west and east Germany. Eur Respir J 1996; 9: 2541-2552

[59] Nikula K], Green FH. Animal models of chronic bronchitis and their relevance to studies of particle-induced disease. Inhal Toxicol 2000; 12 (Suppl. 04): $123-153$

[60] Kodavanti UP, Mebane R, Ledbetter A et al. Variable pulmonary responses from exposure to concentrated ambient air particles in a rat model of bronchitis. Toxicol Sci 2000; 54: 441-451

[61] https://www.baua.de/DE/Angebote/Rechtstexte-und-TechnischeRegeln/Berufskrankheiten/pdf/Merkblatt-4111.pdf?__blob=publicationFile\&v=2. 1997

[62] Bauer H-D. https://publikationen.dguv.de/dguv/pdf/10002/rep0795.pdf. 1995

[63] Marple VA, Rubow KL. An evaluation of the GCA respirable dust monitor 101-1. Am Ind Hyg Assoc J 1978; 39: 17-25

[64] Köhler D, Vastag E. [Bronchial clearance]. Pneumologie 1991; 45 : $314-332$

[65] Oberdörster G. Lung particle overload: implications for occupational exposures to particles. Regul Toxicol Pharmacol 1995; 21: 123-135

[66] Sturm R. A computer model for the clearance of insoluble particles from the tracheobronchial tree of the human lung. Comput Biol Med 2007; 37: $680-690$

[67] Esplugues A, Ballester F, Estarlich M et al. Indoor and outdoor concentrations and determinants of NO2 in a cohort of 1-year-old children in Valencia, Spain. Indoor Air 2010; 20: 213-223

[68] Frey SE, Destaillats H, Cohn S et al. Characterization of indoor air quality and resident health in an Arizona senior housing apartment building. J Air Waste Manag Assoc 2014; 64: 1251 - 1259

[69] Mitteilungen der Ad-hoc-Arbeitsgruppe Innenraumrichtwerte der Innenraumlufthygiene-Kommission des Umweltbundesamtes und der Obersten Landesgesundheitsbehörden. [Health evaluation of fine particulate matter in indoor air]. Bundesgesundheitsblatt Gesundheitsforschung Gesundheitsschutz 2008; 51: 1370-1378

[70] Sahu V, Elumalai SP, Gautam S et al. Characterization of indoor settled dust and investigation of indoor air quality in different microenvironments. Int | Environ Health Res 2018: 28419-28431

[71] Heudorf U. [Particulate matter in classrooms - problem and the impact of cleaning and ventilation with the City of Frankfurt am Main as an example]. Gesundheitswesen 2008; 70: $231-238$

[72] Directive 2008/50/EC of the European Parliament and of the Council on ambient air quality and cleaner air for Europe. 2008. https://eurlex.europa.eu/legal-content/en/ALL/?uri=CELEX\%3A32008L0050

[73] World Health Organization. Air Quality Guideline for Europe. 2000. www.euro.who.int/__data/assets/pdf_file/0005/74732/E71922.pdf

[74] World Health Organization. Air Quality Guidelines for Europe. Global update 2005. Particulate matter, ozone, nitrogen dioxide and sulfur dioxide. 2006: http://www.euro.who.int/__data/assets/pdf_file/ 0005/78638/E90038.pdf 
[75] International Programme on Chemical Savety. Chemical Safety Information from Intergovernmental Organizations. Environmental Health Criteria 188. Nitrogen Oxides. 1997. http://www.inchem. org/documents/ehc/ehc/ehc188.htm

[76] Hasselblad V, Eddy DM, Kotchmar DJ. Synthesis of environmental evidence: nitrogen dioxide epidemiology studies. J Air Waste Manage Assoc 1992; 42: 662-671

[77] Brasche S, Witthauer ], Bischof W et al. [Determination of NO2 exposure - personal passive sampling versus indoor measurement]. Zentralbl Hyg Umweltmed 1998; 201: 229-239

[78] Logue JM, Klepeis NE, Lobscheid AB et al. Pollutant exposures from natural gas cooking burners: a simulation-based assessment for Southern California. Environ Health Perspect 2014; 122: 43-50

[79] Melia RJ, Florey CD, Chinn S et al. Investigations into the relations between respiratory illness in children, gas cooking and nitrogen dioxide in the U.K. Tokai J Exp Clin Med 1985; 10: 375-378

[80] Brand P, Bertram J, Chaker A et al. Biological effects of inhaled nitrogen dioxide in healthy human subjects. Int Arch Occup Environ Health 2016; 89: 1017-1024

[81] Brown JS. Nitrogen dioxide exposure and airway responsiveness in individuals with asthma. Inhal Toxicol 2015; 27: 1-14

[82] Ezratty V, Guillossou G, Neukirch C et al. Repeated nitrogen dioxide exposures and eosinophilic airway inflammation in asthmatics: a randomized crossover study. Environ Health Perspect 2014; 122: $850-855$

[83] Hesterberg TW, Bunn WB, McClellan RO et al. Critical review of the human data on short-term nitrogen dioxide (NO2) exposures: evidence for NO2 no-effect levels. Crit Rev Toxicol 2009; 39: 743 - 781

[84] https://www.nap.edu/catalog/13374/acute-exposure-guideline-levels-for-selected-airborne-chemicals-volume-11. 2012

[85] Dijkstra A, Vonk JM, Jongepier $\mathrm{H}$ et al. Lung function decline in asthma: association with inhaled corticosteroids, smoking and sex. Tho$\operatorname{rax} 2006 ; 61: 105-110$

[86] Harmsen L, Gottlieb V, Makowska Rasmussen L et al. Asthma patients who smoke have signs of chronic airflow limitation before age 45. J Asthma 2010; 47: 362-366

[87] Church DF, Pryor WA. Free-radical chemistry of cigarette smoke and its toxicological implications. Environ Health Perspect 1985; 64: $111-126$

[88] https://www.bundestag.de/blob/559628/ c67c74a62d1a30f6e342462f00c9ce98/wd-8-034-18-pdf-data.pdf. 2018

[89] Perfetti TA, Rodgman A. The Complexity of Tobacco and Tobacco Smoke. Beiträge zur Tabakforschung International/Contributions to Tobacco Research 2014; 24: 215-232

[90] Chen A, Wink DA. Small molecule signaling agents: the integrated chemistry and biochemistry of nitrogen oxides, oxides of carbon, dioxygen, hydrogen sulfide, and their derived species. Chem Res Toxicol 2012; 25: $769-793$

[91] DeMartino AW, Kim-Shapiro DB, Patel RP et al. Nitrite and nitrate chemical biology and signalling. BrJ Pharmacol 2019; 176: 228 - 245

[92] Fukuto JM, Carrington SJ, Tantillo DJ et al. Small molecule signaling agents: the integrated chemistry and biochemistry of nitrogen oxides, oxides of carbon, dioxygen, hydrogen sulfide, and their derived species. Chem Res Toxicol 2012; 16; 25: $769-793$

[93] Lundberg JO, Palm J, Alving K. Nitric oxide but not carbon monoxide is continuously released in the human nasal airways. Eur Respir J 2002; 20: $100-103$

[94] Ford PC, Wink DA, Stanbury DM. Autoxidation kinetics of aqueous nitric oxide. FEBS Lett 1993; 326: 1-3

[95] Frank NR, Yoder RE, Brain JD et al. SO2 (35S labeled) absorption by the nose and mouth under conditions of varying concentration and flow. Arch Environ Health 1969; 18: 315-322
[96] Speizer FE, Frank NR. The uptake and release of $\mathrm{SO} 2$ by the human nose. Arch Environ Health 1966; 12: 725 - 728

[97] Riess U, Tegtbur U, Fauck C et al. Experimental setup and analytical methods for the non-invasive determination of volatile organic compounds, formaldehyde and NOx in exhaled human breath. Anal Chim Acta 2010; 669: 53-62

[98] Hagenbjörk A, Malmqvist E, Mattisson K et al. The spatial variation of $\mathrm{O}(3), \mathrm{NO}, \mathrm{NO}(2)$ and $\mathrm{NO}(\mathrm{x})$ and the relation between them in two Swedish cities. Environ Monit Assess 2017; 189: 161

[99] https://www.umweltbundesamt.de/daten/luft/ozon-belastung. 2018

[100] Rabl P, Scholz W. Wechselbeziehungen zwischen Stickstoffoxid- und Ozon-Immissionen, Datenanalysen aus Baden-Württemberg und Bayern 1990 - 2003. https://www4.lubw.baden-wuerttemberg.de/ servlet/is/14245/entwicklung_stickstoffoxid_immissionen_1995_2003_Kurzfassung.pdf?command=downloadContent\&filename=entwicklung_stickstoffoxid_immissionen_1995_2003_Kurzfassung.pdf. 2017

[101] https://fritzmielert.de/feinstaub/districts/. 2017

[102] https://www.umweltbundesamt.de/daten/luft/luftschadstoff-emissionen-in-deutschland/emission-von-feinstaub-der-partikelgroesse-pm10\#textpart-1. 2018

[103] von Basshuysen R. Ottomotor mit Direkteinspritzung: Verfahren, Systeme, Entwicklung, Potenzial. Wiesbaden: Springer Vieweg; 2013

[104] Merker GP, Teichmann R et al., Hrsg. Grundlagen Verbrennungsmotoren. Funktionsweise und alternative Antriebssysteme Verbrennung, Messtechnik und Simulation. Wiesbaden: Springer Vieweg; 2014

[105] https://www.wkm-ev.de/images/20170708_Die_Zukunft_des_Verbrennungsmotors.pdf. 2017

[106] https://www.adac.de/infotestrat/tests/eco-test/partikel_benziner/ default.aspx. 2017

[107] https://www.adac.de/rund-ums-fahrzeug/abgas-diesel-fahrverbote/dieselkauf-abgasnorm/rde-messungen-cf-faktor/. 2017

[108] Dageförde H, Kubach H, Koch T et al. Innermotorische Ursachen für Partikelemissionen bei Ottomotoren mit Direkteinspritzung. Bargende M, Hrsg. 14. Internationales Stuttgarter Symposium: Automobil- und Motorentechnik. Wiesbaden: Springer Vieweg; 2014: $435-459$

[109] Dageförde H, Bertsch M, Kubach H et al. Reduktion der Partikelemissionen bei Ottomotoren mit Direkteinspritzung. Motortechnische Zeitschrift 2015; 76: 86-93

[110] https://www.umweltbundesamt.de/sites/default/files/medien/360/ dokumente/empfehlungen_und_richtwerte_ergebnisprotokoll_der_7._sitzung_am_3.und4_._mai_2018.pdf. 2018

[111] https://www.umweltbundesamt.de/themen/gesundheit/kommissionen-arbeitsgruppen/ausschuss-fuer-innenraumrichtwerte-vormals-ad-hoc. 2018

[112] https://www.umweltbundesamt.de/sites/default/files/medien/384/ bilder/dateien/2_abb_staub-pm10-emi-quellkat_2018.pdf. 2018

[113] https://www.umweltbundesamt.de/daten/luft/luftschadstoff-emissionen-in-deutschland/stickstoffoxid-emissionen\#textpart-1. 2018

[114] Price RHM, Graham C, Ramalingam S. Association between viral seasonality and meteorological factors. Sci Rep 2019; 9: 929

[115] Schwarz K, Biller H, Windt $\mathrm{H}$ et al. Characterization of exhaled particles from the healthy human lung-a systematic analysis in relation to pulmonary function variables. J Aerosol Med Pulm Drug Deliv 2010; $23: 371-379$

[116] Gralton J, Tovey ER, McLaws ML et al. Respiratory virus RNA is detectable in airborne and droplet particles. J Med Virol 2013; 85: $2151-2159$ 
[117] Yan J, Grantham M, Pantelic J. EMIT Consortium. et al. Infectious virus in exhaled breath of symptomatic seasonal influenza cases from a college community. Proc Natl Acad Sci USA 2018; 115: $1081-1086$

[118] Newman SP, Agnew JE, Pavia D et al. Inhaled aerosols: lung deposition and clinical applications. Clin Phys Physiol Meas 1982; 3: 1-20

[119] Asgharian B, Price OT, Yurteri CU et al. Component-specific, cigarette particle deposition modeling in the human respiratory tract. Inhal Toxicol 2014; 26: 36-47

[120] Kane DB, Asgharian B, Price OT et al. Effect of smoking parameters on the particle size distribution and predicted airway deposition of mainstream cigarette smoke. Inhal Toxicol 2010; 22: 199-209

[121] Sahu SK, Tiwari M, Bhangare RC et al. Particle Size Distribution of Mainstream and Exhaled Cigarette Smoke and Predictive Deposition in Human Respiratory Tract. Aerosol and Air Quality Research 2013; 13: $324-332$

[122] Chepiga TA, Morton MJ, Murphy PA et al. A comparison of the mainstream smoke chemistry and mutagenicity of a representative sample of the US cigarette market with two Kentucky reference cigarettes (K1R4F and K1R5F). Food Chem Toxicol 2000; 38: 949-962

[123] https://monographs.iarc.fr/wp-content/uploads/2018/06/mono100E-6.pdf. 2004

[124] Benjamin RM. Exposure to tobacco smoke causes immediate damage: a report of the Surgeon General. Public Health Rep 2011; 126 : 158 - 159. https://www.ncbi.nlm.nih.gov/pmc/articles/ PMC3056024/

[125] Colpani V, Baena CP, Jaspers L et al. Lifestyle factors, cardiovascular disease and all-cause mortality in middle-aged and elderly women: a systematic review and meta-analysis. Eur J Epidemiol 2018; 33: $831-845$

[126] Sauer WH, Berlin JA, Strom BL et al. Cigarette yield and the risk of myocardial infarction in smokers. Arch Intern Med 2002; 162: 300 306

[127] Inoue-Choi M, Liao LM, Reyes-Guzman C et al. Association of Longterm, Low-Intensity Smoking With All-Cause and Cause-Specific Mortality in the National Institutes of Health-AARP Diet and Health Study. JAMA Intern Med 2017; 177: 87-95

[128] Hansen MS, Licaj I, Braaten T et al. Sex Differences in Risk of Smoking-Associated Lung Cancer: Results From a Cohort of 600,000 Norwegians. Am J Epidemiol 2018; 187: 971 - 981
[129] Harris JE, Thun MJ, Mondul AM et al. Cigarette tar yields in relation to mortality from lung cancer in the cancer prevention study II prospective cohort, 1982-8. BMJ 2004; 328: 72

[130] Lee PN, Forey BA, Coombs KJ. Systematic review with meta-analysis of the epidemiological evidence in the 1900s relating smoking to lung cancer. BMC Cancer 2012; 12: 385

[131] Lee PN, Forey BA, Thornton AJ et al. The relationship of cigarette smoking in Japan to lung cancer, COPD, ischemic heart disease and stroke: A systematic review. F1000Res 2018; 7: 204

[132] Forey BA, Thornton AJ, Lee PN. Systematic review with meta-analysis of the epidemiological evidence relating smoking to COPD, chronic bronchitis and emphysema. BMC Pulm Med 2011; 11: 36

[133] Lee EC, Fragala MS, Kavouras SA et al. Biomarkers in Sports and Exercise: Tracking Health, Performance, and Recovery in Athletes. J Strength Cond Res 2017; 31: 2920-2937

[134] Milara J, Cortijo J. Tobacco, inflammation, and respiratory tract cancer. Curr Pharm Des 2012; 18: 3901 - 3938

[135] Sun Q, Wang A, Jin X et al. Long-term air pollution exposure and acceleration of atherosclerosis and vascular inflammation in an animal model. JAMA 2005; 294: $3003-3010$

[136] Zhang T, Chen Y, Liu H et al. Chronic unpredictable stress accelerates atherosclerosis through promoting inflammation in apolipoprotein E knockout mice. Thromb Res 2010; 126: 386 - 392

[137] Lu XT, Liu YF, Zhang L et al. Unpredictable chronic mild stress promotes atherosclerosis in high cholesterol-fed rabbits. Psychosom Med 2012; 74: 604-611

[138] Mejia-Carmona GE, Gosselink KL, Pérez-Ishiwara G et al. Oxidant/ antioxidant effects of chronic exposure to predator odor in prefrontal cortex, amygdala, and hypothalamus. Mol Cell Biochem 2015; 406: $121-129$

[139] Schneider A, Cyrys J, Breitner S et al. Quantifizierung von umweltbedingten Krankheitslasten aufgrund der Stickstoffdioxid-Exposition in Deutschland. Abschlussbericht im Auftrag des Umweltbundesamtes, überarbeitete Version (Februar 2018). Umweltbundesamt; 2018. ISSN 1862-4340. https://www.umweltbundesamt.de/ sites/default/files/medien/421/publikationen/abschlussbericht_no2_krankheitslast_final_2018_03_05.pdf

[140] Morfeld P, Erren TC. [Why is the "Number of Premature Deaths Due to Environmental Exposures" not Appropriately Quantifiable?] Gesundheitswesen 2019; 81: 144-149 